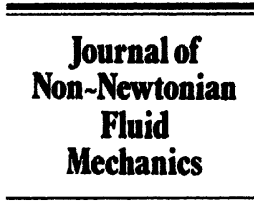

www.elsevier.com/locate/jnnfm

\title{
Asymmetric flows of viscoelastic fluids in symmetric planar expansion geometries
}

\author{
Paulo J. Oliveira* \\ Departamento de Engenharia Electromecânica, Universidade da Beira Interior, 6201-001 Covilhã, Portugal
}

Received 9 April 2003; received in revised form 11 June 2003

\begin{abstract}
The flow of viscoelastic liquids with constant shear viscosity through symmetric sudden expansions is studied by numerical means. The geometry considered is planar and the constitutive model follows the modified FENE-CR equation, valid for relative dilute solutions of polymeric fluids. For Newtonian liquids in a 1:3 expansion we predict the result that the flow becomes asymmetric for a Reynolds number (based on upstream mean velocity and channel height) of about 54, in agreement with previously published results. For the non-Newtonian case the transition depends on both the concentration and the extensibility parameters of the model, and the trend is for the pitch-fork bifurcation to occur at higher Reynolds numbers. Detailed simulations are carried out for increasing Reynolds number, at fixed concentration and Weissenberg number, and for increasing concentration at a fixed Reynolds number of 60 . The results given comprise size and strength of the recirculation zones, bifurcation diagrams, and streamline plots.
\end{abstract}

(C) 2003 Elsevier B.V. All rights reserved.

Keywords: Sudden expansion; Planar expansion; Asymmetric flow; Coanda effect; Viscoelastic fluid; FENE-CR

\section{Introduction}

The flow of viscoelastic liquids through expansion geometries is relevant to a number of practical engineering applications, including extrusion processes, mold filling, processing of food stuffs, creams and pharmaceutical matters, and in many other manufacturing processes. In general, these processes involve the flow of viscoelastic materials through ducts and channels with variable cross-sections and, either in order to achieve specific shapes or for other circumstances, those cross-sections are endowed to change abruptly to larger or smaller areas. The problem of flow through contractions has been much studied, with both Newtonian and non-Newtonian fluids [1-3], but non-Newtonian flow through abrupt expansions has received much less attention. This is particularly true for expansions of the planar type,

\footnotetext{
* Fax: +351-27-532-9972.

E-mail address: pjpo@ubi.pt (P.J. Oliveira).
} 
which are of interest here as they may provoke an asymmetric flow pattern even when the expansion is itself symmetric. It is for this configuration, under laminar flow regime with viscoelastic fluids, that existing published studies are scarce.

A notable exception is the work of Townsend and Walters [4] who have conducted an experimental investigation, based on flow visualization, of the flow of a $0.15 \%$ aqueous solution of polyacrylamide through planar type of expansions, and of a fiber suspension through axisymmetric expansions. Some numerical simulations were also carried out with the PTT model (for the polyacrylamide solution) and the streamlines compared with the visualization results. The main conclusion was that viscoelasticity does indeed lead to a reduction of vortex intensity. For the case which could be considered as two-dimensional, with an expansion ratio of 3:40 $(E \equiv D / d=13.3)$, the Reynolds number covered were too low $(\operatorname{Re} \leq 7)$ for the observation of any steady asymmetric flow pattern. Later, Baloch et al. [5,6] simulated some of the experimental cases in [4] by using again the linear form of the Phan-Thien/Tanner model; these simulations were restricted to high expansion ratios and low Reynolds numbers $(R e \leq 4)$. No attempt was made to capture flow asymmetries.

Representative purely numerical studies of viscoelastic flow through planar expansions $(E=4)$ are those of Darwish et al. [7] and Missirlis et al. [8]; both employ the upper convected Maxwell model (UCM) under creeping flow conditions $(R e=0.2)$ and since flow asymmetries were not expected, only half of the full domain was actually used in these numerical studies (the symmetry assumption is then quite adequate). While the meshes used in [7] were too coarse for quantitative results, the medium meshes of [8] clearly show a reduction in the size of the corner vortex, from $x_{\mathrm{r}} / D=0.15$ at Weissenberg number of $W e=0$ (Newtonian case) to approximately 0 at $W e=1.5$, thus corroborating the experimental findings of Townsend and Walters regarding elimination of recirculation regions by viscoelasticity effects.

Very recently, an experimental work by Poole and Escudier [9] has been published, dealing with turbulent motion of aqueous polymer solutions (0.01- $0.175 \%$ polyacrylamide) in planar expansion with expansion ratio of 1.43. Due to turbulence a number of interesting phenomena arises, which is too vast to be discussed here, but for the rather small expansion ratio considered the flow is more like a backward-facing step flow and asymmetries are not expected, or actually noticed, as commented by the authors. A feature worth noting is that the vortices are seen to increase in size with elasticity (although their intensity still diminishes), contrary to the trend reported by all authors in laminar flows; this must be associated with turbulence effects.

So, we see that much of the works found in the literature are related to very low Reynolds number flows when bifurcation effects are not present. However, for Reynolds numbers of around 50-100, with Re based on the upstream channel height and the average velocity, the flow of Newtonian fluids is known to give rise to an asymmetric pattern, with a larger and a smaller recirculation zone behind the step change. Such phenomenon occurs in planar expansions (it does not occur in axisymmetric expansions) and may be explained by a Coanda effect by which any perturbation of the flow field, pushing the main flow to one of the sides of the expansion, gives rise to larger velocities and lower pressures there, and hence the asymmetry will naturally tend to be accentuated.

Studies of this phenomenon with non-Newtonian fluids could not be found in the specialized literature, except the case of Mishra and Jayaraman [10] who investigated numerically and experimentally the flow of an inelastic power-law fluid in a large expansion ratio duct. Otherwise, all works referred to in the previous paragraphs (except [9]) dealt with symmetrical expanding flows at relatively low Reynolds numbers (below $\approx 10$ ). The purpose of the present study was to ascertain what possible effects could the 
elastic nature of the fluid have on the enhancement, or otherwise, of steady flow bifurcations. We took a sudden expanding channel, with an expansion ratio of $E \equiv D / d=3$, where $D$ is the height of the downstream larger channel and $d$ is the height of the upstream smaller channel. Flows with this particular value of expansion ratio have been subject to a great deal of theoretical, experimental and numerical works with Newtonian fluids [11-13], which are useful for validation. We have then carried out a great number of numerical simulations, with a finite volume method, for a FENE type of constitutive model, and the results of these simulations are here discussed. In Section 2, we present the governing equations, which are solved with the numerical methodology briefly outlined in Section 3. The results are then given in Section 4, which is subdivided into three headings dealing, in succession, with numerical accuracy, validation results for Newtonian fluids, and results for viscoelastic fluid flow. Some conclusions are listed in Section 5.

\section{Flow and constitutive equations}

The problem here considered is the two-dimensional isothermal flow of an incompressible liquid, as it expands from a straight channel of height $d$ to a larger channel of height $D$ (see Fig. 1). This flow is governed by the usual equations of continuity and motion, which can be written in vector form as:

$$
\begin{aligned}
\nabla \cdot \boldsymbol{u} & =0, \\
\rho \frac{D \boldsymbol{u}}{D t} & =-\nabla p+\nabla \cdot \boldsymbol{\tau}_{\mathrm{tot}},
\end{aligned}
$$

where $\boldsymbol{u}$ is the velocity vector, $\rho$ and $p$ are the fluid density (assumed constant) and pressure, respectively, $D / D t \equiv \partial / \partial t+\boldsymbol{u} \cdot \boldsymbol{\nabla}$ is the substantial derivative, and $\boldsymbol{\tau}_{\text {tot }}$ the total extra stress tensor. For a homogeneous solution of a Newtonian solvent and a polymeric solute, the extra stress is decomposed as $\boldsymbol{\tau}_{\text {tot }}=\boldsymbol{\tau}_{\mathrm{s}}+\boldsymbol{\tau}$, with a Newtonian solvent component $\boldsymbol{\tau}_{\mathrm{s}}=\eta_{\mathrm{s}}\left(\boldsymbol{\nabla} \boldsymbol{u}+\boldsymbol{\nabla} \boldsymbol{u}^{\mathrm{T}}\right) \equiv 2 \eta_{\mathrm{s}} \boldsymbol{D}$, where the solvent viscosity $\eta_{\mathrm{s}}$ is constant, and a polymeric component $\boldsymbol{\tau}$. To account for elasticity effects, and since we wish to separate such effects from shear-thinning ones, we took a form of constitutive equation for finite extensibility non-linear dumbbells, valid for dilute solutions of polymeric materials, following a proposal by Chilcott

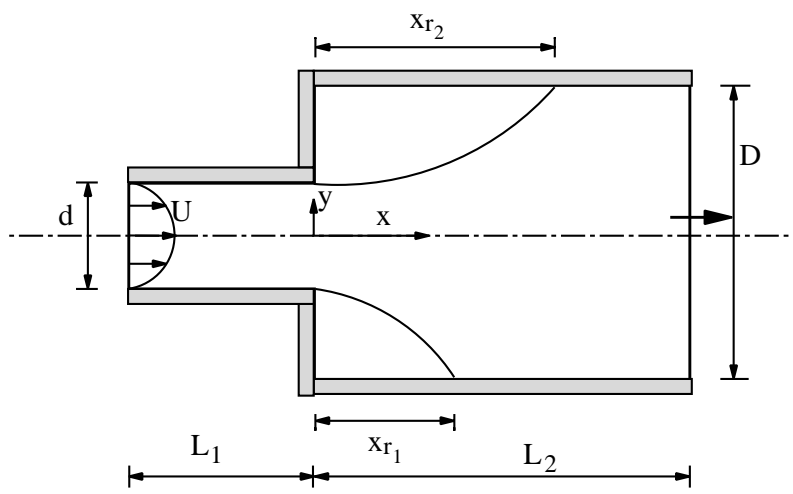

Fig. 1. Sketch of flow configuration. 
and Rallison [14]:

$$
\boldsymbol{\tau}+\frac{\lambda}{g(\tau)} \stackrel{\nabla}{\boldsymbol{\tau}}=2 \eta_{\mathrm{p}} \boldsymbol{D}
$$

with the function $g(\tau)$ expressed by:

$$
g(\tau)=\frac{L^{2}+\left(\lambda / \eta_{\mathrm{p}}\right) \operatorname{tr}(\boldsymbol{\tau})}{L^{2}-3} .
$$

In these equations, $\operatorname{tr}$ is the trace operator, $\lambda$ is a constant relaxation time, $\eta_{\mathrm{p}}$ is the polymer viscosity (also taken as constant) and $L^{2}$ the extensibility parameter that measures the size of the polymer molecule in relation to its equilibrium size. Generally we have $L^{2} \gg 1$ and a typical value may be taken as $L^{2} \approx 100$ [15].

It should be appreciated that this model constitutes an empirical extension of the original FENE-P model of Bird et al. [16] (further improved in Bird et al.'s book [17]) with the purpose of having a constant shear viscosity for a simple shear flow, as discussed in the review of Bird and Wiest [18]. The resulting material functions in steady flows, except for the viscosity, are however not much different in character from those for the FENE-P; the additional simplification embodied in Eq. (3), compared with the exact FENE-CR equation of Chilcott and Rallison, is that $D g / D t$ is assumed to be negligible. Consequently, the constitutive model defined by Eq. (3) and used in the present simulations, is conveniently named a modified Chilcott-Rallison model, FENE-MCR. It has been used by Coates et al. [19] in a numerical study of axisymmetric contraction flows, and also in our previous study of vortex shedding phenomena with viscoelastic fluids [20]. If for time-dependent flows there subsists a difference between the rheological behaviour of the models FENE-CR and FENE-MCR, for steady flows, under both pure shear and pure elongational situations, the material functions of these models are identical. Under simple shear flow (shear rate $\dot{\gamma}$ ), the normal stresses are:

$$
\tau_{x x}=\frac{-L^{2}+\sqrt{L^{4}+8 \dot{\gamma}^{2} \lambda^{2}\left(L^{2}-3\right)}}{2 \lambda / \eta_{\mathrm{p}}} \quad \text { and } \quad \tau_{y y}=0,
$$

which enable calculation of the first normal stress coefficient,

$$
\Psi_{1} \equiv \frac{\tau_{x x}-\tau_{y y}}{\dot{\gamma}^{2}},
$$

while the shear viscosity is constant and is given by:

$$
\eta(\dot{\gamma})=\eta_{\mathrm{s}}+\eta_{\mathrm{p}} \equiv \eta_{0} .
$$

A convenient measure of polymer concentration is the solvent viscosity ratio defined as $\beta \equiv \eta_{\mathrm{s}} / \eta_{0}$. Under uniaxial extensional flow the elongational viscosity $\eta_{\mathrm{E}} \equiv\left(\tau_{z z}-\tau_{r r}\right) / \dot{\epsilon}(\dot{\epsilon}$ is the elongation rate $)$ is determined from:

$$
\eta_{\mathrm{E}}=3 \eta_{\mathrm{s}}+\eta_{\mathrm{p}}\left(\frac{2}{1-(2 \lambda \dot{\epsilon} / g)}+\frac{1}{1+(\lambda \dot{\epsilon} / g)}\right),
$$

where the unknown $g$ in Eq. (8) has the same meaning of function $g$ in Eq. (4) and is obtained here from solution of the following cubic equation:

$$
\left(L^{2}-3\right) g^{3}-\left[(\lambda \dot{\epsilon})\left(L^{2}-3\right)+L^{2}\right] g^{2}-\left[2(\lambda \dot{\epsilon})^{2}\left(L^{2}-3\right)-(\lambda \dot{\epsilon}) L^{2}+6(\lambda \dot{\epsilon})^{2}\right] g+2(\lambda \dot{\epsilon})^{2} L^{2}=0 .
$$




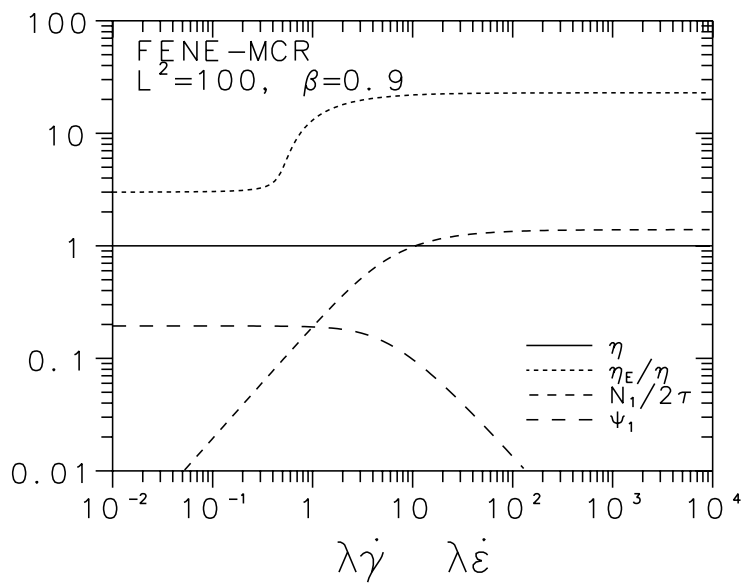

Fig. 2. Rheometrical properties of the FENE-MCR (or FENE-CR) model in steady shear and extensional flows.

The various rheometrical functions of interest here are represented in Fig. 2 and their behaviour will be useful in the discussion of the results given in Section 4.

For convenience, a summary of the main governing equations requiring solution by means of the numerical methodology to be described in the next section is given below, under non-dimensional form:

$$
\begin{aligned}
& \frac{D \boldsymbol{u}}{D t}=-\nabla p+\frac{\beta}{\operatorname{Re}} \nabla^{2} \boldsymbol{u}+\frac{1-\beta}{\operatorname{Re}} \nabla \cdot \boldsymbol{\tau}, \\
& \boldsymbol{\tau}+\frac{W e}{g(\tau)} \frac{D \tau}{D t}=\left(\nabla \boldsymbol{u}+\nabla \boldsymbol{u}^{\mathrm{T}}\right)+\frac{W e}{g(\tau)}\left(\boldsymbol{\tau} \cdot \nabla \boldsymbol{u}+\nabla \boldsymbol{u}^{\mathrm{T}} \cdot \boldsymbol{\tau}\right),
\end{aligned}
$$

where the index $\mathrm{T}$ denotes the transpose operator. The continuity equation remains as in Eq. (1) while the $g$ function (Eq. (4)) is now written as:

$$
g(\tau)=\frac{L^{2}+W e \operatorname{tr}(\boldsymbol{\tau})}{L^{2}-3} .
$$

Although not explicitly denoted, all variables in the flow (Eq. (10)) and the constitutive (Eq. (11)) equations above are non-dimensionalized with a velocity scale $U$ (average velocity in small channel), a length scale $d$ (height of small channel), a time scale $d / U$, a pressure scale $\rho U^{2}$, and an elastic stress scale $\eta_{\mathrm{p}} U / d$. The relevant dimensionless groups to be varied in a parametric way are the extensibility parameter of the FENE-CR model, $L^{2}$, the solvent viscosity ratio,

$$
\beta=\frac{\eta_{\mathrm{s}}}{\eta_{0}}
$$

the Reynolds number,

$$
R e=\frac{\rho U d}{\eta_{0}},
$$

and the Weissenberg number:

$$
W e=\frac{\lambda U}{d} .
$$


In order to avoid any unnecessary confusion, in the next sections, all the variables are written in dimensional form and their non-dimensional form will be explicitly indicated.

\section{Numerical methodology}

We follow the finite volume method explained in detail in [21] and only a few general comments are in order here. A key feature of the method of [21] is that it uses a collocated mesh, meaning that all dependent variables are evaluated and stored at the center of the control volumes (cells) composing the computational mesh. As a consequence, special procedures are required to ensure pressure/velocity coupling (following the Rhie and Chow [22] method) and velocity/stress coupling (following the Oliveira et al. [21] method). Here, we shall not dwell in these techniques, as they are sufficiently documented in the specialized literature [3,20,21,23]. A brief description of the solution algorithm follows.

The stress equations (11) are discretized, by integration over the control volumes (volume $V$ ), and are linearized and written under the form:

$$
a_{\mathrm{P}}^{\tau} \boldsymbol{\tau}_{\mathrm{P}}=\sum_{F=1}^{6} a_{F}^{\tau} \boldsymbol{\tau}_{F}+S_{\boldsymbol{\tau}},
$$

with coefficients $a_{F}^{\tau}$ based on convective fluxes calculated with the upwind scheme, and source term $\boldsymbol{S}_{\boldsymbol{\tau}}$ containing contributions from velocity-gradient terms in the Oldroyd derivative of $\boldsymbol{\tau}(\boldsymbol{\tau}$ in Eq. (3)), inertial term and deferred-correction terms from representation of the convective fluxes with the high-order scheme CUBISTA [24]. This is a formally third-order convection scheme in smooth flows (possessing mild gradients) which was shown in [24] to have improved iterative convergence properties when employed in conjunction with implicit solvers, as it is the case here. Furthermore, there is ample evidence that viscoelastic flow calculations require accurate discretization of the convection fluxes in the constitutive equations, even for low Reynolds number flows (the Weissenberg number might be high), and the plain upwind scheme is too inaccurate.

In step 1 of the algorithm, the above sets of linear equations, for each stress component $\tau_{i j}$ Eq. (16), are solved sequentially by a bi-conjugate gradient method. Solution for a steady state is achieved by a pseudo time-marching technique whereby the time step $\delta t$ acts as an inertial under-relaxation term. Once updated stress fields are obtained from solution of Eq. (16), the discretized momentum conservation equations can be solved implicitly for the intermediate velocity $\boldsymbol{u}^{*}$ (step 2 of the algorithm):

$$
a_{\mathrm{P}} \boldsymbol{u}_{\mathrm{P}}^{*}=\sum_{F=1}^{6} a_{F} \boldsymbol{u}_{F}^{*}+S_{\boldsymbol{u}} .
$$

The coefficients now contain diffusive and convective fluxes; the former are evaluated with the second-order central differencing scheme, and the latter with the formally third-order CUBISTA scheme, as noted above, which is implemented through the deferred correction technique. There are two important source terms here, to be included into $S_{u}$ : the stress divergence term $S_{u}(\tau)$, and the pressure gradient term $S_{u}(\nabla p)$; the origin of these terms is immediate from Eq. (10).

While the stress divergence term is evaluated based on stresses obtained from step 1 of the algorithm, but taking into account the stress/velocity problem described in [21], the pressure gradient term follows a standard pressure-correction technique used in classical Newtonian CFD. The SIMPLEC algorithm for 
time marching calculation is here followed [25,26]. The source term $S_{u}(\nabla p)$ is evaluated with pressure values from the previous time step $\left(S_{u}\left(\nabla p^{*}\right)\right)$ and velocity and pressure corrections are then imposed (step 3 of the algorithm):

$$
\begin{aligned}
& \boldsymbol{u}=\boldsymbol{u}^{*}-\frac{\nabla p^{\prime}}{\rho V / \delta t}, \\
& p=p^{*}+p^{\prime},
\end{aligned}
$$

where the asterisk denotes existing (in storage) values and $\delta t$ is the time step. This entails solution of a Poisson-like equation for the pressure correction $p^{\prime}$, having the form:

$$
a_{\mathrm{P}}^{p} p_{\mathrm{P}}^{\prime}=\sum_{F=1}^{6} a_{F}^{p} p_{F}^{\prime}-\left(\nabla \cdot \boldsymbol{u}^{*}\right),
$$

with coefficients given by $a_{\mathrm{f}}^{p}=B_{\mathrm{f}}^{2} /(\overline{\rho V / \delta t})_{\mathrm{f}}$ and $a_{\mathrm{P}}^{p}=\sum a_{F}^{p}$ ( $B_{\mathrm{f}}$ is a cell-face surface area and the overbar denotes linear interpolation). Note that the source term in the pressure correction Eq. (20) is a mass source (the divergence of the intermediate velocity field), based on the velocity resulting from the momentum equations, which should tend to zero when the velocity field will simultaneously satisfy the momentum and continuity equations.

With the new values of $\boldsymbol{\tau}, \boldsymbol{u}$ and $p$, from Eqs. (16), (18) and (19), the whole procedure can then be restarted at a new time level, and repeated until the residuals of the governing equations fall below a prescribed tolerance (TOL). Here, we used TOL $=10^{-4}$, based on normalized values (normalization factors: mass - inlet mass flow rate; momentum - inlet mass flow times characteristic inlet velocity $U$; stress-viscosity times characteristic velocity $U$ divided by inlet channel height $d$ ).

In broad terms, the algorithm just described corresponds to a simplified form of that given in [20] and is adequate for the simulation of steady-state flows. While it is expected that the bifurcation phenomenon here considered is of steady-state nature, any unsteady manifestation would be felt by an impossibility to converge, to the prescribed tolerance, the whole set of equations involved. Such occurrence was not manifested during the current simulations.

\section{Results}

All results given in this section pertain to an expansion ratio of $E=3$ and the flow geometry is depicted in Fig. 1 where some of the notation is defined. The reason for choosing $E=3$ lies on the many numerical and theoretical results existing for that particular expansion ratio [10-13], for Newtonian fluids, against which we can compare our results (Section 4.2).

The inlet to the flow domain is located some distance $\left(L_{1}\right)$ upstream of the expansion plane (this is taken as the coordinate origin, $x=0)$, while the outlet is at an even longer distance $\left(L_{2}\right)$ from that plane. Guided upon the study of Hawa and Rusak [13] who recommended $L_{1} \geq 2$ and $L_{2} \geq 40$ for $10 \leq R e \leq 100$, and also on simulations of tubular Newtonian laminar flow by Oliveira and Pinho [27] who observed that the upstream effect of expansion was not felt at a distance longer than about 1 diameter (for $R e \gtrsim 10$ ), we chose $L_{1}=2$ and $L_{2}=50$ (all distances are normalized with $d$ ). The Reynolds number is here defined with the average incoming velocity, see Eq. (14), but it is noted that many authors prefer to use the maximum inlet velocity, $R e_{0} \equiv \rho U_{0} d / \eta_{0}$. Since at the inlet we have imposed a fully 
developed profile, which is parabolic for the axial velocity component, those two Reynolds numbers are related by $R e_{0}=1.5 R e$. For the FENE-MCR (or the FENE-CR) the fully developed velocity and shear stress distributions in channel flow are identical to those for a Newtonian fluid, given by:

$$
u=1.5 U\left(1-\left(\frac{y}{d / 2}\right)^{2}\right)
$$

and

$$
\tau_{x y}=-\left(\frac{12 \eta_{\mathrm{p}} U}{d}\right)\left(\frac{y}{d}\right),
$$

while the axial polymer normal stress is given by:

$$
\tau_{x x}=2 \eta_{\mathrm{p}} \lambda \frac{\dot{\gamma}^{2}}{g},
$$

with the local shear rate obtained from

$$
\dot{\gamma}=-\frac{12 U y}{d^{2}},
$$

and where the function $g$ is calculated from the following expression:

$$
g=\frac{L^{2}+\sqrt{L^{4}+8\left(L^{2}-3(\lambda \dot{\gamma})^{2}\right)}}{2\left(L^{2}-3\right)} .
$$

Since FENE-type models do not predict a non-vanishing second normal stress coefficient, the lateral normal stress in fully developed flow is zero:

$$
\tau_{y y}=0 .
$$

Notice that if the dumbbells are allowed to stretch infinitely, $L^{2} \rightarrow \infty$, then Eq. (25) gives $g \rightarrow 1$, and the normal stress distribution from (23) becomes equal to the solution for the Oldroyd-B fluid in a channel.

At outlet, the imposed boundary conditions were of vanishing axial variation for all quantities, $\partial / \partial x \equiv 0$, except pressure which was linearly extrapolated from the inside. These conditions are adequate and do not affect the main flow characteristics near the expansion, provided $L_{2}$ is sufficiently long.

This section is arranged as follows. First, we give some details about the computational meshes here utilized and, based on results on these meshes, we quantify the numerical accuracy. Then, we give the results for the Newtonian case with the main purpose of validating the calculations. Obviously, this could not be done with viscoelastic fluids because, as discussed in the Introduction, we could not find any adequate data set from the literature. Finally, we consider the viscoelastic case, for which we study the effects of elasticity, extensibility, polymer concentration and inertia.

\subsection{Meshes and quantification of accuracy}

Three computational meshes have been employed in this study and their main characteristics are given in Table 1, which includes the total number of control volumes (NC) and of degrees-of-freedom (DOF), the minimum cell size ( $\delta x_{\min }$, equal to $\delta y_{\min }$; values are normalized with $d$ ) and the expansion factor for 
Table 1

Main characteristics of computational meshes

\begin{tabular}{lrrll}
\hline & NC & DOF & $\delta x_{\min }$ & $f_{x}$ \\
\hline Mesh-1 & 1550 & 9300 & 0.10 & 1.0754 \\
Mesh-2 & 6200 & 37200 & 0.05 & 1.0370 \\
Mesh-3 & 24800 & 148800 & 0.025 & 1.0183 \\
\hline
\end{tabular}

the cell size along the $x$-direction. Most calculations to be presented used the medium mesh (Mesh-2), and the fine (Mesh-3) and coarse (Mesh-1) meshes were obtained by doubling or halving, respectively, the number of cells along the $x$ - and $y$-directions, so as to enable quantification of numerical accuracy through application of Richardson's extrapolation technique. Mesh spacing is uniform in the lateral $y$-direction and non-uniform along the streamwise $x$-direction, with higher concentration of cells around the expansion plane $(x=0)$, as shown by Fig. 3 (medium Mesh-2). From that plane the cell spacing expands at a constant geometric rate of $3.7 \%$ for Mesh-2 (so $\delta x_{i+1}=f_{x} \delta x_{i}$, where $i$ denotes a given $x$-position). Compared with other works, the present level of mesh refinement is similar to that used by Hawa and Rusak [13] (their base mesh had $\delta x=0.05$ ) who solved the Newtonian problem with a vorticity/stream function formulation using a finite-difference method; in relation to works dealing with viscoelastic flow simulations in planar expansions at very low $R e$, Mesh-2 is much finer than those previously used $[5,7,8]$.

Numerical accuracy was assessed for both Newtonian and viscoelastic fluids with a Reynolds number of 60 , in the middle of the range here considered $(R e=0.01-100)$ but for which the flow is already asymmetric for both fluid types. We used the streamwise sizes of the vortices formed behind either step side of the expansion, and their intensity expressed in terms of recirculating flow rate scaled with inlet flow rate, as solution functionals from which numerical accuracy is quantified. These results obtained on the three meshes are given in Table 2, for the Newtonian and the viscoelastic flow cases $\left(R e=60, L^{2}=100\right.$, $\beta=0.9$ and $W e=2$ ). The basic idea of Richardson's extrapolation is to assume that any point-value of the solution, or any solution functional, $\phi$, converges with mesh refinement as $\phi=\phi_{h}+C h^{p}$, where $h$ is the mesh size (our $\delta x$ ), $C$ is a constant, $p$ is the order of the scheme, and $\phi_{h}$ the value of $\phi$ obtained on mesh $h$. If we denote the cell size on Mesh-3 as $h$ and since we have performed mesh halving, then the cell size on Mesh- 2 is $2 h$ and the corresponding discretization errors will be $\epsilon_{h}=\Delta \phi /\left(2^{p}-1\right)$ on Mesh-3 and $\epsilon_{2 h}=2^{p} \Delta \phi /\left(2^{p}-1\right)$ on Mesh-2 $\left(\Delta \phi \equiv \phi_{h}-\phi_{2 h}\right)$. By assuming second-order accuracy $(p=2)$, based on previous works with the same code [20,23,28], the extrapolated values (to a zero mesh size) are calculated from $\phi=\left(4 \phi_{h}-\phi_{2 h}\right) / 3$ and are given in Table 2 for the vortices sizes $\left(X_{\mathrm{r}}\right)$ and intensities

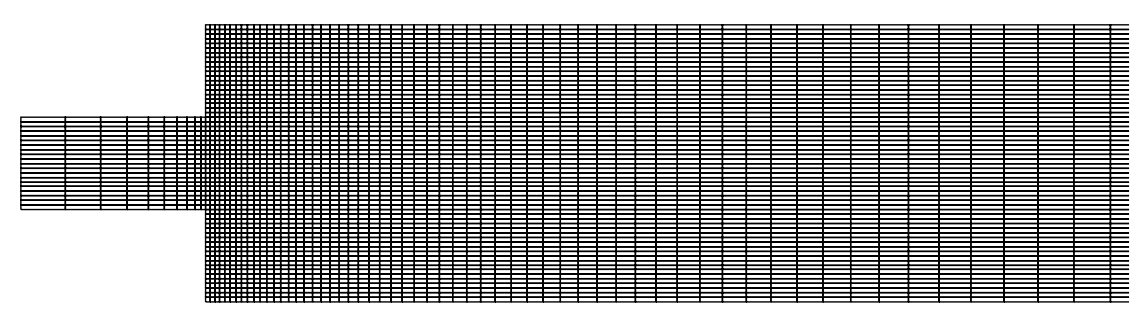

Fig. 3. Zoomed view of Mesh-2 $(-2 \leq x \leq 10,-1.5 \leq y \leq+1.5)$. 
Table 2

Effect of mesh refinement: (a) Newtonian; (b) viscoelastic

\begin{tabular}{lllll}
\hline & $X_{\mathrm{r} 1}$ & $X_{\mathrm{r} 2}$ & $\psi_{\mathrm{r} 1}\left(\times 10^{-2}\right)$ & $\psi_{\mathrm{r} 2}\left(\times 10^{-2}\right)$ \\
\hline (a) Newtonian & & & & 6.760 \\
Mesh-1 & 3.9012 & 7.5851 & 5.709 & 6.780 \\
Mesh-2 & 3.9326 & 7.6085 & 5.721 & 6.857 \\
Mesh-3 & 3.9337 & 7.6223 & 5.725 & 6.872 \\
Richardson's extrapolation & 3.9341 & 7.6269 & 0.28 & 0.90 \\
Discretization error (\%) & 0.04 & 0.24 & & 6.291 \\
(b) Viscoelastic & & & 5.327 & 6.427 \\
Mesh-1 & 4.124 & 7.241 & 5.391 & 6.537 \\
Mesh-2 & 4.092 & 7.241 & 5.485 & 6.574 \\
Mesh-3 & 4.082 & 7.254 & 5.916 & 1.7 \\
Richardson extrapolation & 4.079 & 7.258 & 1.7 & \\
Discretization error $(\%)$ & 0.33 & 0.33 & & \\
\hline
\end{tabular}

$\left(\psi_{\mathrm{r}}\right)$ (denoted "Richardson's extrapolation" in the table). The discretization errors on Mesh-2 (our base mesh for the remaining results) are also given in the table.

It is seen that the discretization errors for the recirculation size lie below $0.5 \%$ for both the Newtonian and viscoelastic cases, while errors in recirculation intensity rise to about $1 \%$ for the Newtonian fluid and $2 \%$ for the FENE-MCR fluid. The fact that errors in $\psi_{\mathrm{r}}$ are higher than errors in $X_{\mathrm{r}}$ was expected because evaluation of $\psi_{\mathrm{r}}$ requires integration of the resulting velocity field and that tends to lower the accuracy (see discussion in [28] where further references on this issue are given). In conclusion, our results are accurate to within $\approx 1 \%$, with even better accuracy in results that are directly evaluated from the numerical solution $(\lesssim 0.5 \%)$ and some deterioration of accuracy for integrated results (up to $2 \%$ for viscoelastic fluids).

\subsection{Results for the Newtonian case (validation)}

In Fig. 4, we compare the predicted bifurcation results (vortex size asymmetry $D X \equiv\left(X_{\mathrm{r} 2}-X_{\mathrm{r} 1}\right)$ versus $R e$ ) with those of Fearn et al. [11], who actually measured the recirculation lengths for increasing Reynolds numbers and who have also carried out simulations with numerical bifurcation techniques and a finite element method. Other authors [10] have considered the 1:3 expansion as well, and have demonstrated close matching with the data of Fearn et al. It is seen from Fig. 4 that, for the Newtonian case, there is very good agreement amongst the results of these various sources. Such level of agreement is remarkable as it would appear that the exact "location" of the bifurcation, in terms of Reynolds number, would be rather sensitive to a number of variables, as mesh resolution, numerical precision, initial conditions, triggering mechanism, etc. In most earlier calculations of these phenomena, the asymmetric flow was usually triggered by introducing a slight geometrical asymmetry in the symmetric configuration, or by adding a small perturbation to the velocity profile imposed at inlet, or to the whole initial velocity field $[10,11,13,29,30]$. In fact, we found that such artificial triggering devices are not required and in our case the numerical solution naturally evolved to an asymmetric flow when the Reynolds number was above the critical value, $R e_{\mathrm{c}}$. All the computations of Fig. 4 have been started from an initial uniform velocity 


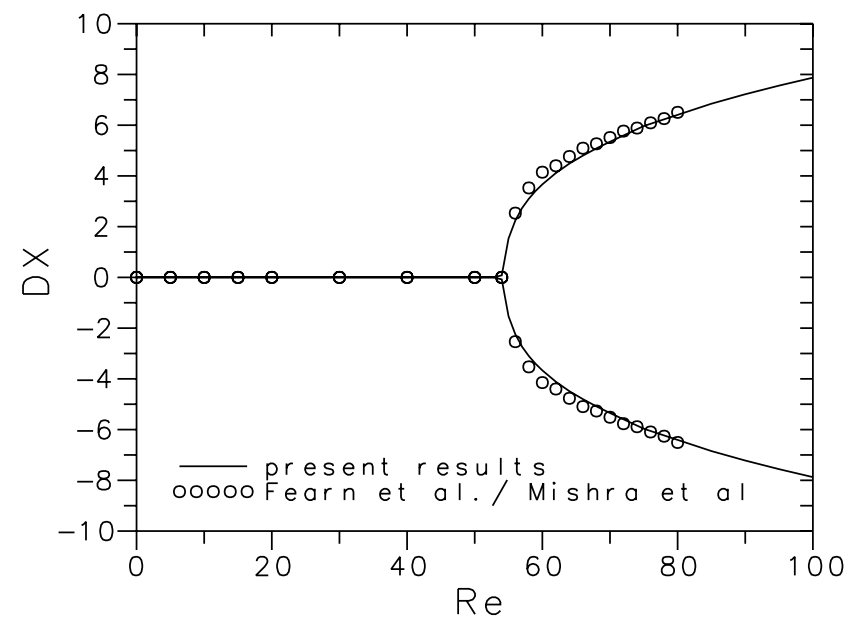

Fig. 4. Comparison of the predicted bifurcation diagram for Newtonian flow with the experimental data from Fearn et al. [11] (numerical results of Mishra and Jayaraman [10] closely follow that data).

field (with the average velocities for each channel segment, that is $U \equiv U_{1}=Q / d$ and $U_{2}=Q / D$, for the upstream and downstream channels, respectively, where $Q$ is the volumetric flow rate per unit width). This was done on purpose to see how the time marching (iterative convergence) behaviour would be affected by the onset of asymmetric flow conditions. Obviously, the computing times per run are thus much higher than strictly required had the usual continuation technique been used, by restarting a run from an initial field corresponding to the case of the Reynolds number immediately below.

Our data are given in Table 3 . For $R e$ in the range where we knew that bifurcation would occur (guided from the literature) we varied $R e$ by a single unit; outside that range, $R e$ was varied by steps of 5 units. We found a critical Reynolds number of $R e_{\mathrm{c}}=54$; this is, again, in remarkable agreement with other sources where different numerical methods were utilized: Fearn et al. [11] found $R e_{\mathrm{c}}=$ 53.9 with a finite element method; Drikakis [12] found $R e_{\mathrm{c}}=53.3\left(R e_{0}=80\right)$, with fourth-order finite-difference methods; Hawa and Rusak [13] found $R e=53.8$, with linear stability analysis and a vorticity/stream function finite-difference formulation; Mishra and Jayaraman [10] found $R e_{\mathrm{c}}=54$, with a continuation-perturbation approach and a finite element code. Note that we have not tried to be precise on the location of $R e_{\mathrm{c}}$ : it lies somewhere between $R e=53$ and 54. For $R e=54$, the asymmetric state has been clearly achieved; for $R e=53$, although a slight asymmetry is already present, it is so small that cannot be seen in the bifurcation graph of Fig. 4. The numerical behaviour of the time marching algorithm around the bifurcation point is illustrated by Fig. 5 which shows the residuals of the $v$-momentum equation as the simulation time proceeds. These residuals $\left(\ell_{1}\right.$-norm of the discretized equations, conveniently normalized) should essentially tend to zero as a steady-state solution is approached; the prescribed stopping criterion was for a relative tolerance of $10^{-4}$. This value was checked from the computations to be adequate; we monitored not only the residuals of the various equations $\left(u, v\right.$, continuity, $\left.\tau_{x x}, \tau_{y y}, \tau_{x y}\right)$ and values of the fields at a given sensitive point (near one of the reentrant comers), but also the sizes and intensities of the two main recirculation zones. When all residuals were below $10^{-4}$, none of those variables were seen to vary significantly. For some runs, for example, for $R e=30$ (viscoelastic case, given later) and in order to check whether the slight asymmetry seen in $X_{\mathrm{r}}$ and $\psi_{\mathrm{r}}$ would not eventually disappear, we pursued 
Table 3

Predicted vortex data for the Newtonian case

\begin{tabular}{rllll}
\hline$R e$ & $X_{\mathrm{r} 1}$ & $X_{\mathrm{r} 2}$ & $\psi_{\mathrm{r} 1}\left(\times 10^{-2}\right)$ & $\psi_{\mathrm{r} 2}\left(\times 10^{-2}\right)$ \\
\hline 0.1 & 0.523 & 0.523 & 0.0548 & 0.0548 \\
1.0 & 0.570 & 0.570 & 0.0781 & 0.0781 \\
10 & 1.211 & 1.211 & 1.115 & 3.115 \\
20 & 2.111 & 2.111 & 3.059 & 4.059 \\
30 & 3.080 & 3.080 & 4.428 & 5.233 \\
40 & 4.075 & 4.075 & 5.233 & 5.748 \\
50 & 5.080 & 5.081 & 5.748 & 5.826 \\
52 & 5.279 & 5.285 & 5.825 & 5.913 \\
54 & 5.445 & 5.523 & 5.893 & 6.276 \\
56 & 4.440 & 6.678 & 5.745 & 6.553 \\
58 & 4.107 & 7.208 & 5.717 & 6.804 \\
60 & 3.609 & 5.708 & 7.462 \\
64 & 3.935 & 8.246 & 5.712 & 8.567 \\
70 & 3.762 & 9.019 & 5.740 & 9.471 \\
75 & 3.669 & 9.620 & 5.766 & 10.11 \\
80 & 3.651 & 10.06 & 5.794 & 10.69 \\
90 & 3.658 & 10.52 & 5.813 & 11.19 \\
95 & 3.679 & 10.93 & 5.836 & 11.62 \\
100 & 3.708 & 11.30 & 5.853 & 11.98 \\
\hline
\end{tabular}

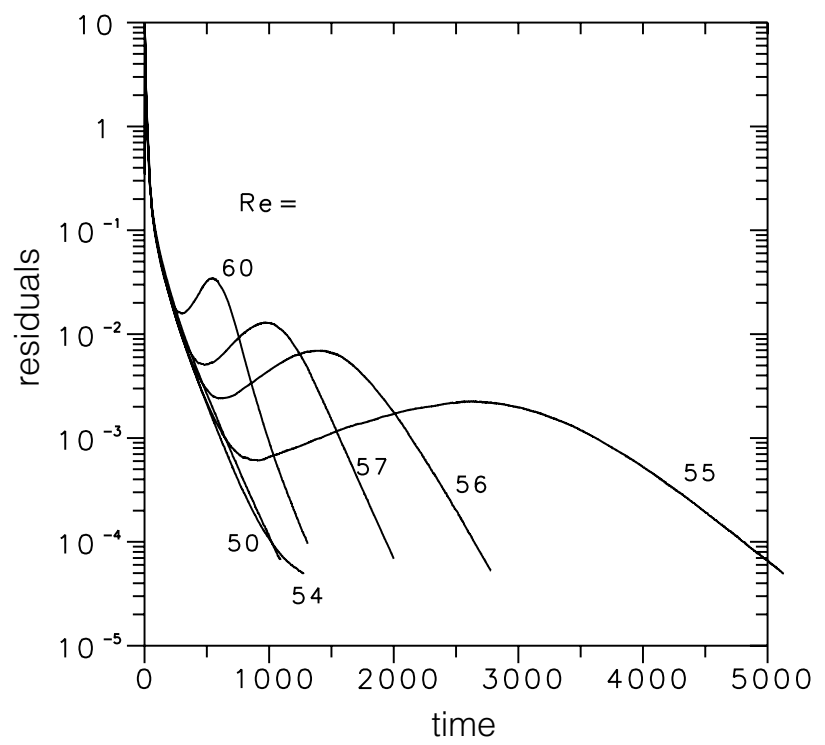

Fig. 5. Residuals history ( $v$-momentum equation) for the Newtonian fluid case. 
the simulations up to a tolerance of $10^{-6}$; the results remained practically identical to those for the lower tolerance of $10^{-4}$.

What is interesting from Fig. 5 is that the residuals decay is qualitatively similar to the results of Hawa and Rusak [13] (their Figs. 14 and 18) where the growth rate of disturbances was tracked; as the critical $R e$ is approached, the convergence to the steady solution becomes more and more difficult to achieve, taking a lot more number of time steps, although it is always monotonic (the residuals decay clearly); for $R e$ above the critical value the number of time steps for convergence actually decreases as $R e$ gets away from $R e_{\mathrm{c}}$, but the convergence is not monotonic. There is an evident reduction of the residuals during the initial stage of the simulation, while the flow remains symmetric, the residuals then increase for a certain duration of simulation time, reflecting the onset of the asymmetric flow situation, and finally there is again an evident reduction of residuals which may take more or less time, typical of iterative finite volume methods as the long-wave numerical errors are reduced and swept out of the domain. We find interesting that this pattern of residuals behaviour (which is just an empirical observation) closely resembles (certainly qualitatively) the results of the stability analysis of [13] for this problem.

A detailed field comparison is now considered. Fearn et al. [11] made some velocity measurements for this flow geometry at the 1:3 expansion ratio. We chose their case $R e=80$, which corresponds to an asymmetric flow situation, and present in Fig. 6 is a comparison between our predictions (the solid lines) and their measurements (the round symbols), at four axial stations situated at $x / d=1.25,5,10$ and 20. There is clearly a very satisfactory agreement amongst these local velocity data.

As a further check for the same expansion ratio $E=3$, we compare our predictions against those of Da Zilwa et al. [30] for the case $R e=187\left(R e_{0}=280\right)$, when a second bifurcation occurs and a new recirculation zone appears by the wall adjacent to the smaller comer vortex. The situation is then as depicted in Fig. 7. Da Zilwa et al. quote the following values for the sizes of the various vortices: $X_{\mathrm{r} 1}=5.0, X_{\mathrm{r} 2}=15.2, X_{\mathrm{r} 3}=12.6$ and $X_{\mathrm{r} 4}=26$. Our simulations are illustrated by the streamline plot of Fig. 7 and gave the results: $X_{\mathrm{r} 1}=4.5, X_{\mathrm{r} 2}=15.1, X_{\mathrm{r} 3}=12.4$ and $X_{\mathrm{r} 4}=25.3$. Thus, a relative good agreement is again observed, even if due account is taken of the inaccuracies inherent to the numerical predictions.

All these results and comparison with existing experimental or numerical data from the literature, for Newtonian fluids, give sufficient confidence on our predictive code allowing us to proceed to the non-Newtonian viscoelastic simulations, for which no adequate data for comparison/validation are available. It is emphasized that the same code was used for both Newtonian and non-Newtonian simulations. In the former, we set the relaxation time to zero, the solvent viscosity to zero, and the polymer viscosity to the required value for a given Reynolds number. In this way, even for a Newtonian simulation, a stress equation separated from the momentum equations was being solved.

\subsection{Results for the viscoelastic case}

For non-Newtonian viscoelastic flows, even for the simple constitutive model here considered, the number of independent parameters that can be varied goes from a single one in the Newtonian case $(R e)$, up to four with the FENE-MCR $\left(R e, W e, L^{2}\right.$ and $\beta$ ), and so a detailed study of all possibilities goes well beyond the present attempt. We have therefore decided to fix all parameters except one at some typical values, which are often used in relation to FENE type of models, and vary separately the remaining parameter. Such separated effects are discussed under different headlines in this section. 

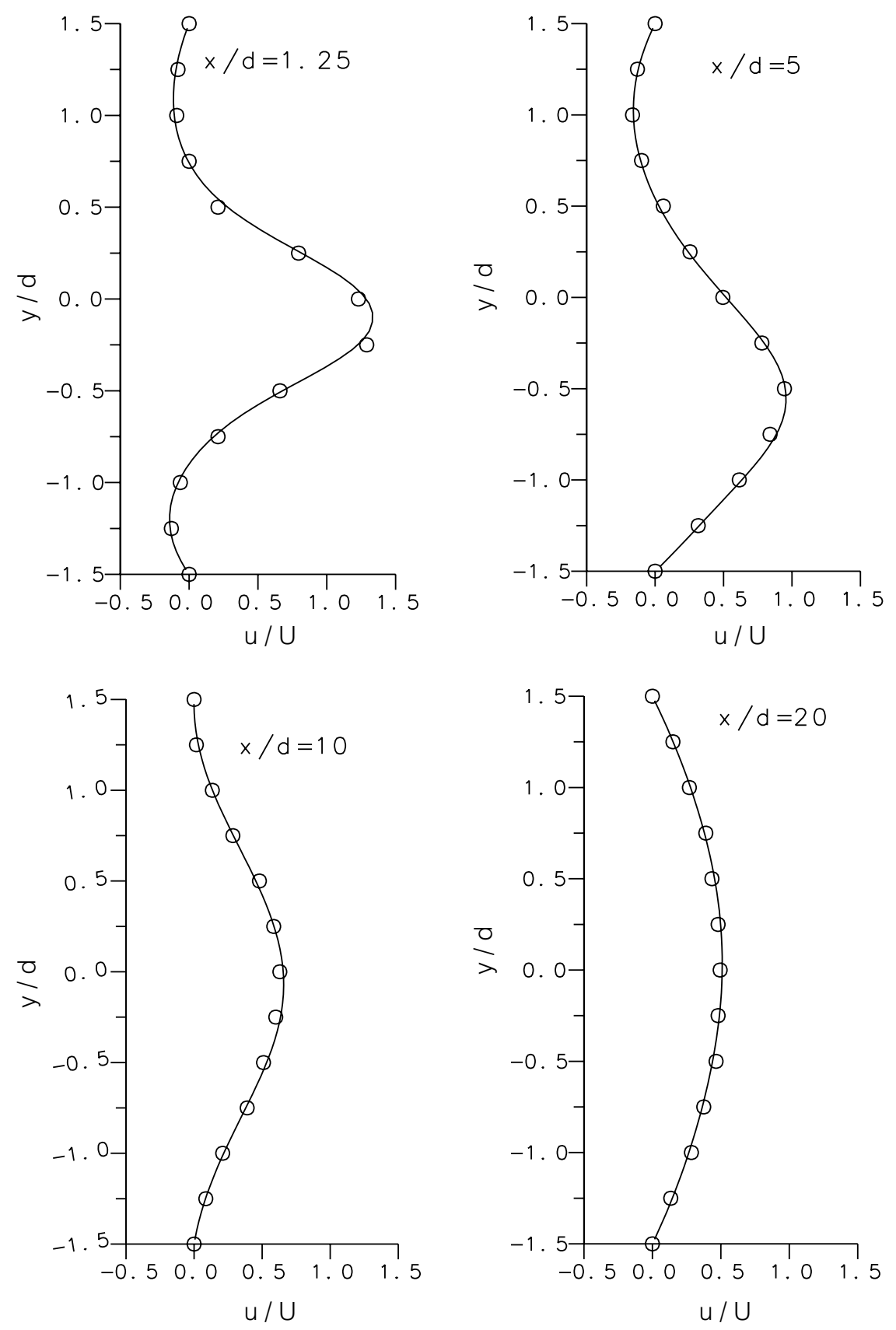

Fig. 6. Detailed comparison of predicted velocity profiles with the measurements of Fearn et al. [11].

\subsubsection{Effect of elasticity, We}

As base values we took the extensibility parameter as $L^{2}=100$, and the concentration parameter close to $c \approx 0.1$, giving $\beta=0.9$. We recall that $L$ is the ratio of the linear size of a dumbbell, the basic unit in FENE models, to its size at equilibrium. So the larger is $L^{2}$, the more can the molecule be extended. For 


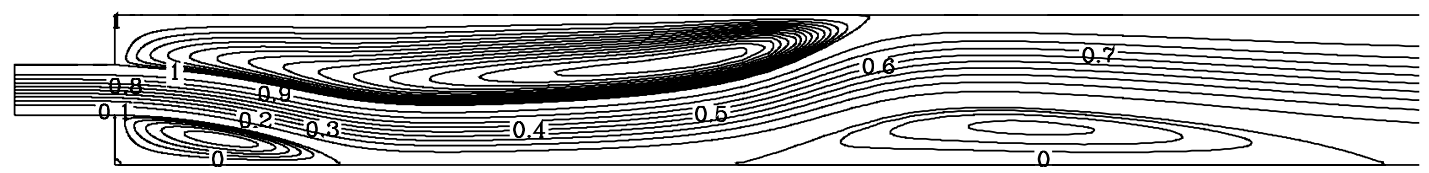

Fig. 7. Illustration of second bifurcation for Newtonian flow, at $R e=187$. Reattachment and detachment lengths of vortices long bottom wall are denoted $X_{\mathrm{r} 1}, X_{\mathrm{r} 3}, X_{\mathrm{r} 4}$; on top wall $X_{\mathrm{r} 2}$.

the particular case of the well-known Oldroyd-B model, an infinite extension is allowed $\left(L^{2} \rightarrow \infty\right)$, but that gives rise to singularity situations in simple extensional flows. With FENE models, $L^{2}$ (or the related $b$ parameter [16]) are typical around 100 [15,16,19], and can be even lower, of order O(10) [31].

The concentration parameter is defined as $c \equiv \eta_{\mathrm{p}} / \eta_{\mathrm{s}}$ and for dilute solutions a typical value is $c \approx 0.1$ [15]. Alternatively, a solvent viscosity ratio can be defined $\beta \equiv \eta_{\mathrm{s}} / \eta$ with $\eta=\eta_{\mathrm{p}}+\eta_{\mathrm{s}}$ being the solution (solvent plus solute) viscosity, having a zero shear rate value of $\eta_{0}$. For the FENE-CR-type model utilized here, $\eta$ is constant (does not depend on shear rate, $\eta \equiv \eta_{0}$ ) and $\beta$ (Eq. (13)) is related to $c$ by $\beta=1 /(1+c$ ); hence $c \approx 0.1$ gives $\beta \approx 0.9$, our base value.

For a concentration of around 0.1 , the velocity field is not expected to be much affected by elasticity (dilute flow regime, [15]). This is borne out in Fig. 8 where the size of the recirculation eddies is represented as a function of the Weissenberg number, at a Reynolds number of $R e=60$. In the previous section, it was shown that for this Reynolds number the stable asymmetric states are already well established in Newtonian flow, but yet that value is not too far away from the critical Reynolds number of $R e_{\mathrm{c}}=54$. Therefore, this level of $R e$ is convenient to ascertain whether elasticity (measured by $W e$ ), at constant concentration $(\beta=0.9)$ and extensibility $\left(L^{2}=100\right)$, may affect the flow to such extent that, for example, the asymmetry might be removed. The results in Fig. 8, given quantitatively in Table 4, show that as We is increased from $W e=0$, the larger of the two corner vortices tends to be reduced in size, while the shorter vortex tends to be elongated. Thus, both effects act to diminish the asymmetry of the flow, but the asymmetry remains for the whole range of We here tested $(W e=0-100)$.

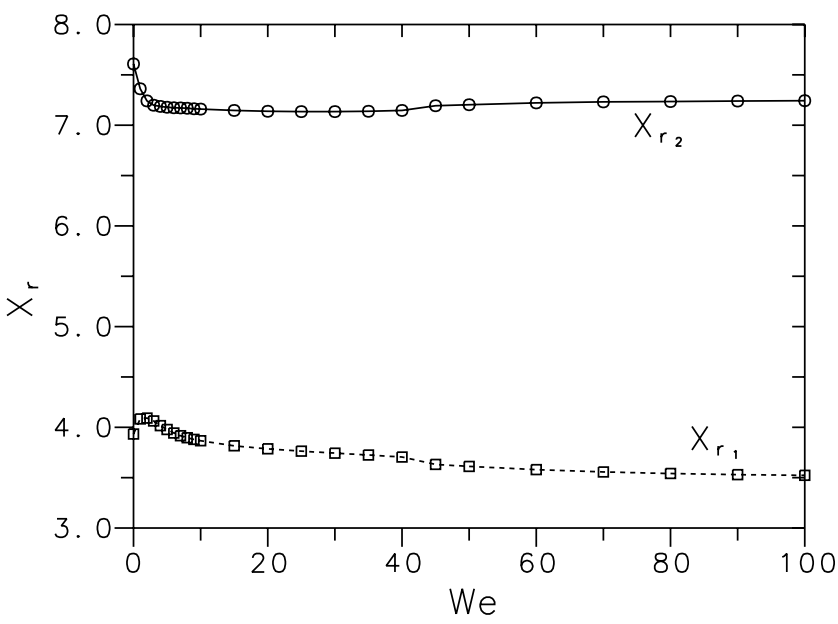

Fig. 8. Effect of elasticity (Weissenberg number) on the size of the two asymmetric vortices $\left(\operatorname{Re}=60, L^{2}=100, \beta=0.9\right)$. 
Table 4

Predicted vortex data for the viscoelastic case-effect of elasticity through $W e\left(R e=60, \beta=0.9, L^{2}=100\right)$

\begin{tabular}{rllll}
\hline$W e$ & $X_{\mathrm{r} 1}$ & $X_{\mathrm{r} 2}$ & $\psi_{\mathrm{r} 1}\left(\times 10^{-2}\right)$ & $\psi_{\mathrm{r} 2}\left(\times 10^{-2}\right)$ \\
\hline 0 & 3.93 & 7.61 & 5.71 & 6.81 \\
1 & 4.08 & 7.36 & 5.50 & 6.52 \\
2 & 4.09 & 7.24 & 5.39 & 6.43 \\
3 & 4.06 & 7.20 & 5.32 & 6.43 \\
4 & 4.02 & 7.19 & 5.26 & 6.46 \\
5 & 3.98 & 7.18 & 5.21 & 6.49 \\
10 & 3.87 & 7.16 & 5.05 & 6.57 \\
15 & 3.82 & 7.15 & 4.99 & 6.60 \\
20 & 3.79 & 7.14 & 4.97 & 6.62 \\
30 & 3.74 & 7.14 & 5.00 & 6.64 \\
40 & 3.70 & 7.15 & 5.08 & 6.66 \\
50 & 3.61 & 7.20 & 5.44 & 6.71 \\
60 & 3.58 & 7.22 & 5.59 & 6.71 \\
70 & 3.56 & 7.23 & 5.67 & 6.72 \\
80 & 3.54 & 7.24 & 5.73 & 6.72 \\
90 & 3.53 & 5.77 & 6.72 \\
100 & 3.52 & 7.24 & 5.80 & 6.72 \\
\hline
\end{tabular}

It is also apparent that, for such low level of concentration $(c \approx 0.1)$, the main influence of We is restricted to Weissenberg numbers in the range $\approx 1-5$, and for higher We there is almost no further effect. Such behaviour is consistent with the rheometric data for the FENE-MCR given in Fig. 2. The initial $(W e=1)$ rapid variation of $X_{\mathrm{r}}$ correlates with the sharp increase in elongational viscosity at $\lambda \dot{\epsilon} \sim 1$ in Fig. 2. For $W e>\approx 20-40$ the vortex sizes remain approximately unchanged, in line with the fully established shear stress ratio seen in Fig. 2 for $\lambda \dot{\gamma} \gtrsim 40$.

\subsubsection{Effect of extensibility, $L^{2}$}

Compared with the Oldroyd-B model, or the related upper convected Maxwell model (the UCM), the FENE-CR offers the possibility of varying the dumbbells extensibility. This may not only be advantageous to better fit rheological data of a given liquid, but it is also welcome in numerical terms; generally, FENE models are not as problematic numerically as the models cited above.

Here, we decided to increase $L^{2}$ from the base value of 100 up to 500 (fixed $R e=60, \beta=0.9$ as before, and $W e=1$ ); the main results are summarized in Table 5. Basically, it is observed that the larger

Table 5

Predicted vortex data for the viscoelastic case—effect of extensibility parameter $L^{2}(W e=1, \beta=0.9, R e=60)$

\begin{tabular}{lllll}
\hline$L^{2}$ & $X_{\mathrm{r} 1}$ & $X_{\mathrm{r} 2}$ & $\psi_{\mathrm{r} 1}\left(\times 10^{-2}\right)$ & $\psi_{\mathrm{r} 2}\left(\times 10^{-2}\right)$ \\
\hline 100 & 4.09 & 7.36 & 5.50 & 6.52 \\
200 & 4.11 & 7.37 & 5.37 & 6.46 \\
300 & 4.14 & 7.39 & 5.27 & 6.40 \\
400 & 4.17 & 7.42 & 5.20 & 6.39 \\
500 & 4.15 & 7.46 & 5.12 & 6.42 \\
\hline
\end{tabular}


Table 6

Predicted vortex data for the viscoelastic case-effect of concentration parameter $\beta\left(W e=2, L^{2}=100, R e=60\right)$

\begin{tabular}{lllll}
\hline$\beta$ & $X_{\mathrm{r} 1}$ & $X_{\mathrm{r} 2}$ & $\psi_{\mathrm{r} 1}\left(\times 10^{-2}\right)$ & $\psi_{\mathrm{r} 2}\left(\times 10^{-2}\right)$ \\
\hline 0.9 & 4.092 & 7.241 & 5.39 & 6.43 \\
0.8 & 4.516 & 6.703 & 5.13 & 5.87 \\
0.7 & 5.447 & 5.723 & 5.05 & 5.16 \\
0.6 & 5.416 & 5.435 & 4.78 & 4.79 \\
0.5 & 5.249 & 5.262 & 4.42 & 4.43 \\
0.4 & 5.043 & 5.046 & 3.86 & 3.86 \\
0.3 & 4.769 & 4.802 & 2.83 & 2.84 \\
\hline
\end{tabular}

vortex tends now to increase, while the smaller vortex remains approximately constant, but the effect is rather small. Even for the case of $W e=2$ not shown in the table, the effect of increasing $L^{2}$ was seen to remain small.

\subsubsection{Effect of concentration, $\beta$}

Besides its physical meaning, the parameter $c$ rules the extent of the coupling between the motion and the constitutive equations. The larger the value for $c$, the more coupled are those equations (following the notion introduced by Patankar [32], the larger is the two-way coupling between kinematics and stress) and, as a consequence, the more difficult is the numerical solution of the underlying flow problem. As mentioned before, $c \approx 0.1$ is typical of dilute polymer solutions, while $c \approx 1.0$ is typical of more concentrated semi-dilute solutions, but it should be remarked that so-called Boger fluids (highly elastic, constant viscosity liquids) are often modeled with $c$ as large as 1 or 8 , giving the values $\beta=0.5$ or $1 / 9$. For the present study, and since our purpose is to ascertain the possible influence of "elasticity" on the Coanda effect with a relatively mobile polymer solution, we varied $\beta$ from 0.9 to 0.3 , at fixed $R e=60$, $L^{2}=100$ and $W e=2$. This latter value was chosen on the basis of Fig. 8 where it is seen that for $W e=2$ the influence of elasticity is almost fully established.

The results of the present parametric study, in terms of vortex size and strength, are given in Table 6 and illustrated in Fig. 9. The effect is for a decreasing $\beta$ to stabilize the flow until a stable symmetrical state is reached for $\beta \leq 0.6$, at $\operatorname{Re}=60$, while the flow is asymmetrical for $\beta>0.6$. Compared with the parameter $L^{2}$ of the previous subsection, it is quite evident that concentration exerts a much stronger influence on flow stability and flow pattern. Fig. 10 compares the resulting streamlines for $\beta=0.9$ and 0.6 (viscoelastic fluid) and the Newtonian case, at $R e=60$. It is clear that the Newtonian flow has the largest asymmetry, this asymmetry is somewhat reduced with a small introduction of elasticity in the fluid, and it is completely attenuated when the concentration of elastic liquid is further increased. To account for the effect of concentration, and in fact to absorb it as a viscoelastic effect, it would be better to measure elasticity by a modified Weissenberg number $W e^{\prime} \equiv\left(\lambda-\lambda_{\mathrm{r}}\right) U / d$, where $\lambda_{\mathrm{r}}$ is the so-called retardation time; that is, $W e^{\prime}=W e(1-\beta)$. Now, when $\beta$ is reduced from 0.9 to $0.3, W e^{\prime}$ suffers a sevenfold increase, thus explaining the added stability of the flow for the symmetrical base states.

As noted above, at the beginning of this sub-section, numerical difficulties in solving the governing equations are expected to be enhanced when $\beta$ is lowered, thus effectively increasing the elasticity of the FENE-CR liquid. This was in fact felt during the current simulations. For example, the cases considered here with $0.7 \geq \beta \geq 0.4$ were started from an initial uniform field with a time step of $\delta t=0.0325$ 


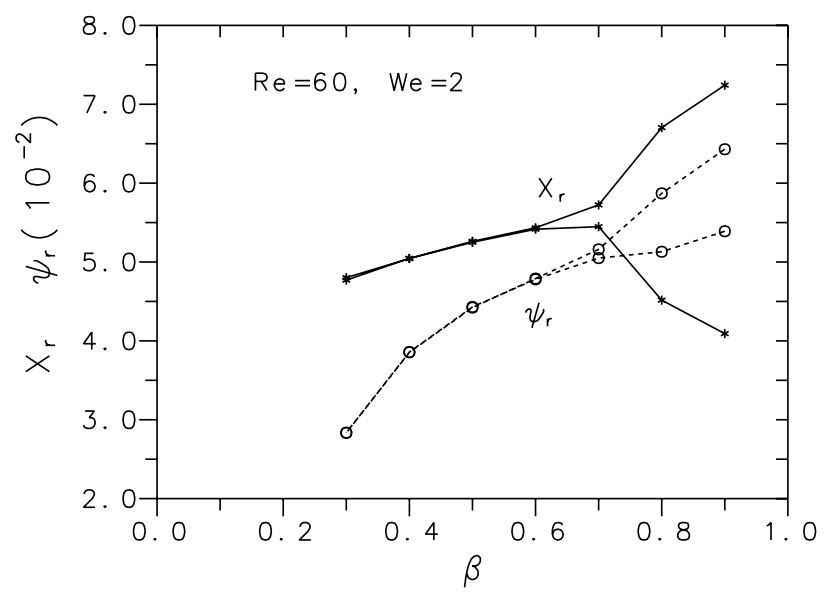

Fig. 9. Effect of concentration upon size and intensity of vortices $\left(\beta \equiv \eta_{\mathrm{s}} / \eta_{0}=1 /(1+c)\right)$.

(non-dimensional) and would converge without problems; but for $\beta=0.3$ it was necessary to use a lower time step $(\delta t=0.016)$ during the initial stage of the simulation in order to procure iterative convergence. On the other hand, for larger $\beta$, the $\delta t$ could be even higher: $\delta t=0.0625$ at $\beta=0.8 ; \delta t=0.125$ at $\beta=0.9$; and $\delta t=0.5$, and often 2 , at $\beta=0$ (Newtonian runs). It should be said that for the range

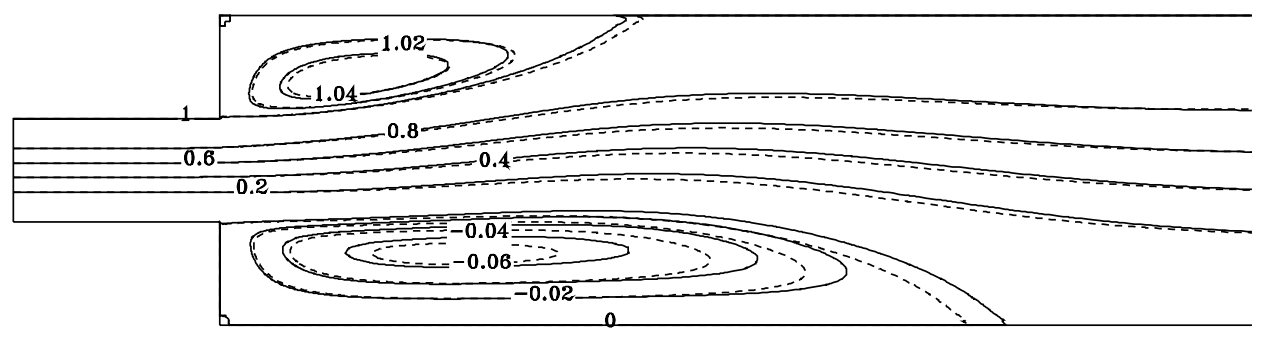

(a)

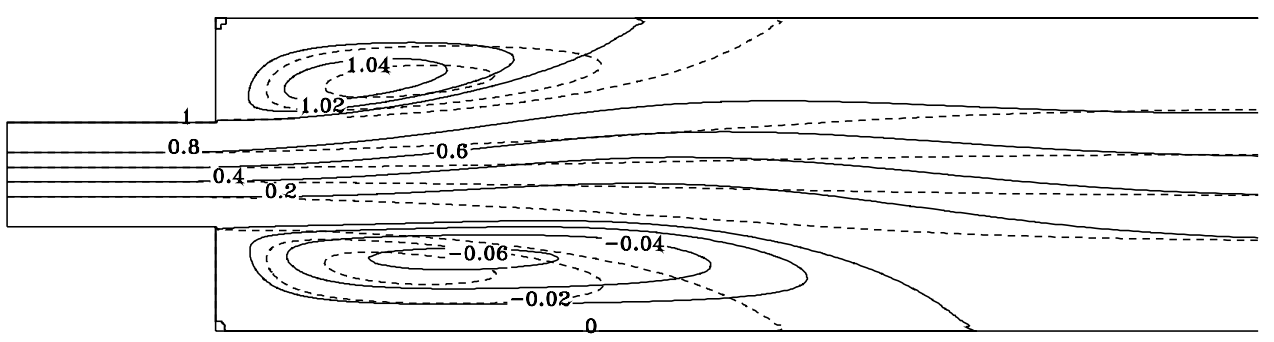

(b)

Fig. 10. Streamline patterns at $R e=60$ : (a) comparison of Newtonian (solid lines) and viscoelastic (dashed lines) case (We $=2$, $L^{2}=100, \beta=0.9$ ); (b) influence of concentration in viscoelastic flow, $\beta=0.9$ (solid lines) and $\beta=0.6$ (dashed lines) (We $\left.=2, L^{2}=100\right)$. 


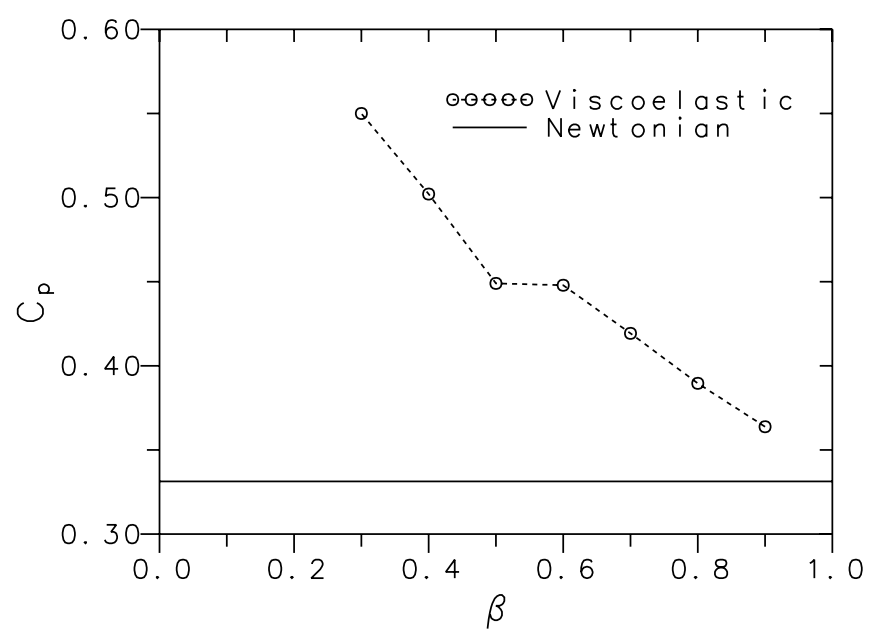

Fig. 11. Variation of the overall pressure drop coefficient $\left(C_{\mathrm{p}}=\left(p_{1}-p_{2}\right) /\left((1 / 2) \rho U^{2}\right)\right)$ with the solvent viscosity ratio $\left(\beta=\eta_{\mathrm{s}} / \eta_{0}\right)$.

of parameters covered in this study, steady (well converged) solutions could always be achieved by an adequate choice of $\delta t$. It may very well be that, for higher elasticity, limits in the maximum attainable We will emerge, as it is common in viscoelastic flow computations.

An interesting effect of polymer concentration is illustrated in Fig. 11 which shows the overall pressure drop coefficient across the expansion $\left(C_{\mathrm{p}} \equiv\left(p_{1}-p_{2}\right) /(1 / 2) \rho U^{2}\right.$, where $p_{1}$ and $p_{2}$ are the pressures acting at the inlet and outlet planes, respectively) as a function of the solvent viscosity ratio $\beta$, for $L^{2}=100, R e=60$ and $W e=2$. The pressure drop diminishes as $\beta$ increases (lower concentration), eventually tending to the Newtonian value for a zero concentration $(\beta \rightarrow 1)$. However, there is a manifest discontinuity in $C_{\mathrm{p}}$ at $\beta \simeq 0.5-0.6$, when the asymmetrical flow state sets in, and it is clear that the pressure drop is higher for the bifurcated state than it would be for a corresponding symmetrical state. Bifurcation is therefore accompanied by an increased pressure loss.

\subsubsection{Effect of inertia, Re}

The main results concerning the viscoelastic flow simulations are given in this subsection which deals with the variation of vortex characteristics with the Reynolds number, for typical values $L^{2}=100$, $W e=2$ and a moderate concentration giving $\beta=0.5$. For example, the MIT group have used a mixture of $0.31 \mathrm{wt}$ \% PIB polymer with a highly viscous Newtonian solvent $(\mathrm{PB}+\mathrm{C} 14)$, thus forming a Boger fluid, which was then represented with several constitutive equations having a solvent viscosity ratio of $\beta=0.59$, and an extensibility of $L^{2}=100$, in a study of flow around cylinder arrays [33]. Such fluid can be taken as representative of the one modeled in the current simulations.

Our numerical data are given in Table 7 and are plotted in Fig. 12(a), which shows the variation of the lengths of the upper and lower vortices with the Reynolds number and in Fig. 12(b), which shows the corresponding recirculation flow rates (scaled with the inlet value). For a Reynolds number up to the critical value $R e_{\mathrm{c}}=64$ the flow remains steady and symmetric, while for larger $R e$ the flow is still steady but asymmetric with a larger and a smaller recirculation length. In Fig. 13 the bifurcation plot for the viscoelastic liquid, $D X \equiv\left(X_{\mathrm{r} 2}-X_{\mathrm{r} 1}\right)$ versus $R e$, is compared with that for the Newtonian case. From 
Table 7

Predicted vortex data for the viscoelastic case-effect of Reynolds number $\left(W e=2, L^{2}=100, \beta=0.5\right.$ )

\begin{tabular}{|c|c|c|c|c|}
\hline$R e$ & $X_{\mathrm{r} 1}$ & $X_{\mathrm{r} 2}$ & $\psi_{\mathrm{r} 1}\left(\times 10^{-2}\right)$ & $\psi_{\mathrm{r} 2}\left(\times 10^{-2}\right)$ \\
\hline 0.01 & 0.394 & 0.396 & 0.0176 & 0.0177 \\
\hline 0.1 & 0.551 & 0.563 & 0.0423 & 0.0476 \\
\hline 1.0 & 0.559 & 0.568 & 0.0452 & 0.0500 \\
\hline 10 & 0.714 & 0.718 & 0.125 & 0.132 \\
\hline 20 & 1.389 & 1.390 & 0.931 & 0.934 \\
\hline 30 & 2.307 & 2.313 & 2.039 & 2.041 \\
\hline 40 & 3.299 & 3.303 & 2.952 & 2.953 \\
\hline 50 & 4.280 & 4.281 & 3.731 & 3.732 \\
\hline 60 & 5.249 & 5.262 & 4.420 & 4.430 \\
\hline 62 & 5.445 & 5.458 & 4.534 & 4.542 \\
\hline 64 & 5.648 & 5.697 & 4.560 & 4.585 \\
\hline 66 & 4.661 & 6.712 & 4.198 & 5.363 \\
\hline 67 & 4.475 & 6.974 & 4.143 & 5.616 \\
\hline 68 & 4.344 & 7.188 & 4.119 & 5.861 \\
\hline 69 & 4.255 & 7.368 & 4.106 & 6.067 \\
\hline 70 & 4.175 & 7.553 & 4.094 & 6.303 \\
\hline 72.5 & 4.046 & 7.936 & 4.084 & 6.817 \\
\hline 75 & 3.966 & 8.284 & 4.092 & 7.350 \\
\hline 80 & 3.877 & 8.903 & 4.124 & 8.283 \\
\hline 85 & 3.849 & 9.450 & 4.165 & 9.073 \\
\hline 90 & 3.848 & 9.942 & 4.207 & 9.741 \\
\hline 95 & 3.863 & 10.38 & 4.246 & 10.28 \\
\hline 100 & 3.888 & 10.79 & 4.282 & 10.76 \\
\hline
\end{tabular}

these figures, two facts can be extracted.

1. The onset Reynolds number for the transition from a symmetric to an asymmetric state is delayed to higher values, specifically from $R e_{\mathrm{c}}=54$ (Newtonian) to $R e_{\mathrm{c}}=64$ (viscoelastic)—elasticity is therefore a stabilizing factor for the occurrence of bifurcation, under laminar flow conditions.

2. Vortex sizes and intensities are smaller for the viscoelastic liquid, compared with the Newtonian, a finding also reported in previous studies under different conditions $[4,5,8]$. This effect is observed for the whole range of $R e$, from 0 to 100 (the exception being the size of the smaller vortex $X_{\mathrm{r} 2}$ which is actually larger for the non-Newtonian fluid) and can be seen as a swelling-like phenomenon similar to that occurring in extrusion processes.

Fig. 14 presents streamline plots of the viscoelastic flow for various Reynolds numbers, from $R e=0.01$, where a bulging of the streamlines at the expansion entrance is clearly seen, to $R e=100$, where the larger vortex is already longer than $10 d$ (the size of the part of the full domain shown in these plots). It is also demonstrated that at $R e=64$ the flow is still essentially symmetric, but at $R e=66$ it is plainly asymmetric. Note that the streamlines inside the recirculation regions are equally spaced but the spacing is not the same for the whole range of Reynolds numbers: $\delta \psi=0.02 \times 10^{-2}$ for $\operatorname{Re} \leq 10 ; \delta \psi=1 \times 10^{-2}$ for $R e \geq 20$, with the innermost curves having $\psi= \pm 0.5 \times 10^{-2}$. This was done to avoid cluttering the figure with too many lines inside the vortices. Values of $\psi$ are normalized with the inlet flow rate so that, along the streamwise direction, $\psi$ varies from 0 to 1 . 


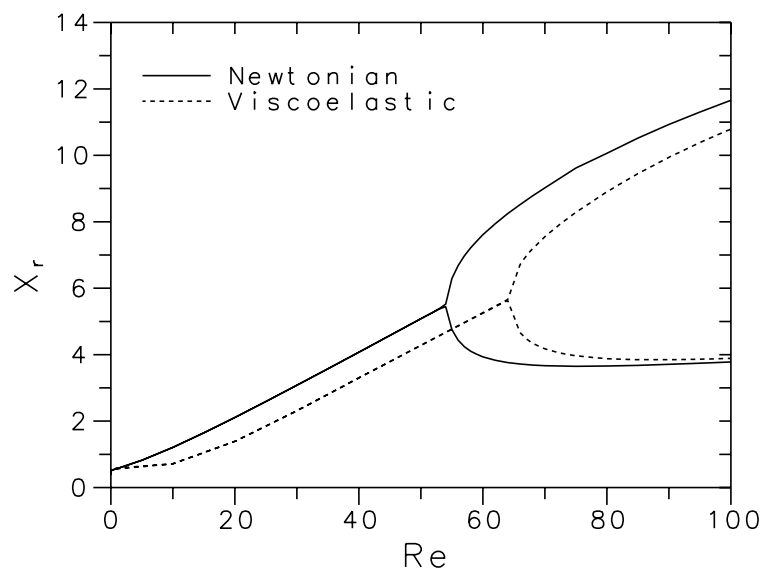

(a)

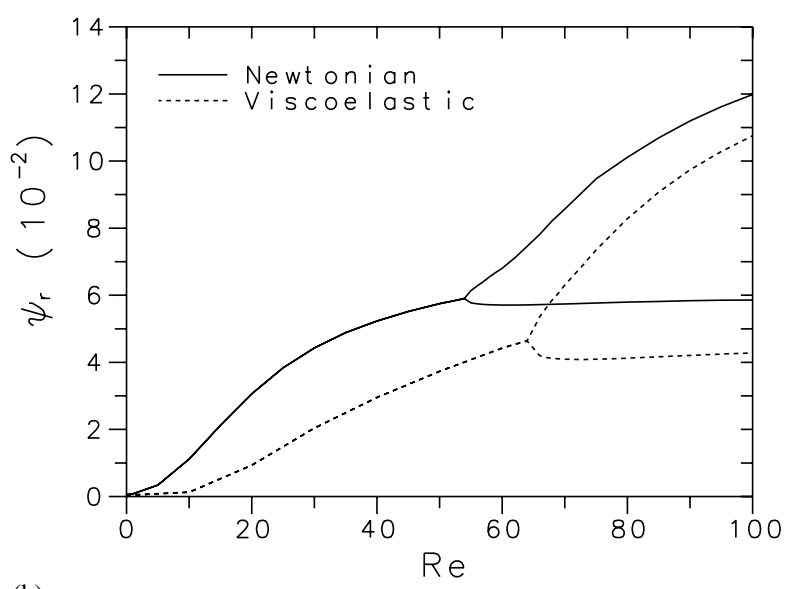

(b)

Fig. 12. Comparison of the predicted vortex characteristics with the Reynolds number, for Newtonian and viscoelastic fluids ( $L^{2}=100$, We $=2, \beta=0.5$ ): (a) size of vortices; (b) intensity of vortices.

For Reynolds numbers above 100, a second pitch-fork bifurcation should occur, with the appearance of a third recirculation zone adjacent to the channel wall containing the smaller corner vortex, similarly to that found for Newtonian fluids and which was illustrated in Fig. 7. This higher range of Re was not investigated. For low Reynolds numbers (say $R e \lesssim 1-10$ ) the flow is symmetric and is dominated by elastic effects.

In fact, if elasticity is measured by an elastic number defined by $E l \equiv W e / R e$, instead of the actual Weissenberg number which is kept constant during the present parametric study, then it is clear from streamline plots (Fig. 14) that the swelling effect is accentuated as $R e$ diminishes. This is particularly noticeable at low $R e$, where the flow is seen to converge towards the centreline before the expansion, followed by a stronger divergence towards the larger channel walls after the expansion. As a consequence, an overshoot of the streamwise velocity component along the centreline is observed as shown in Fig. 15, where the numerical results for the particular case of $R e=0.1$ are plotted. The overshoot is here of $9 \%$ and, for higher $R e$, no overshoot was observed. 


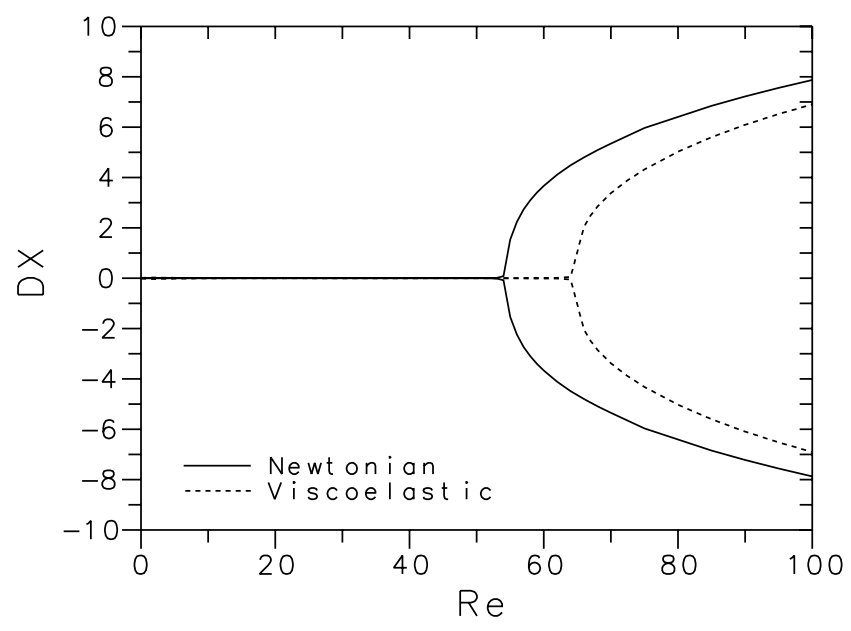

Fig. 13. Bifurcation diagrams for Newtonian and viscoelastic flows in a 1:3 planar expansion. Viscoelastic case at We $=2$, with $L^{2}=100$ and $\beta=0.5$.

For such low levels of Reynolds number, when the flow is essentially symmetric, we are closer to the cases considered by Darwish et al. [7] and Missirlis et al. [8]. Both $X_{\mathrm{r}}$ and $\psi_{\mathrm{r}}$ then tend to constant values, typical of Moffat eddies for the viscoelastic fluid, and these values are not too different from those seen in contraction flows. It should be pointed out, however, that if for a Newtonian fluid the vortices are identical in either contraction or expansion flows, as $R e \rightarrow 0$, the same is not true for viscoelastic fluids on account of history effects through the constitutive equation. This discrepancy between vortex characteristics in contraction and expansion flows should increase as the level of viscoelasticity is increased.

We investigate now the influence of viscoelasticity and inertia on the pressure variation. The total pressure drop normalized with the upstream kinetic energy is represented in Fig. 16 as a function of the Reynolds number for both the Newtonian and the viscoelastic fluid cases. These curves show the typical variation inversely proportional to $R e$, at the low $R e$ range, and tending to a constant value, at high $R e$ for inertia dominated flows. It is also seen that the overall pressure drop is always higher for the viscoelastic fluid (here at $W e=2, \beta=0.5$ and $L^{2}=100$ ) compared with the Newtonian. Since friction losses in straight channels under fully developed conditions for the current constant-viscosity viscoelastic fluid model are identical to the Newtonian losses, the observed higher pressure drop must be associated with a localized loss at the expansion region. In order to understand the causes for this effect, we plot in Fig. 17 the pressure distribution along the channels central plane $(y=0)$ for the Newtonian and viscoelastic cases, and for two typical Reynolds numbers: $R e=50$ (flow is still symmetric) and $R e=100$ (flow is asymmetric). For the flow at higher $R e$ the pressure variation is more complex and reflects the different reattachment lengths of the smaller and the larger asymmetric vortices (inflection seen in the curves). What is however clear from this figure, and is common to its part (a) (for $R e=50$ ) and (b) (for $R e=100)$, is the couple of features that may explain the excess pressure drop of the viscoelastic flow case.

- A history (or memory) effect induces a continuation of the pressure decrease, even after the expansion plane, for the viscoelastic fluid; this results in a lower minimum pressure, occurring at $x>0$.

- The pressure recovery, after the expansion, is lower for the viscoelastic fluid. 

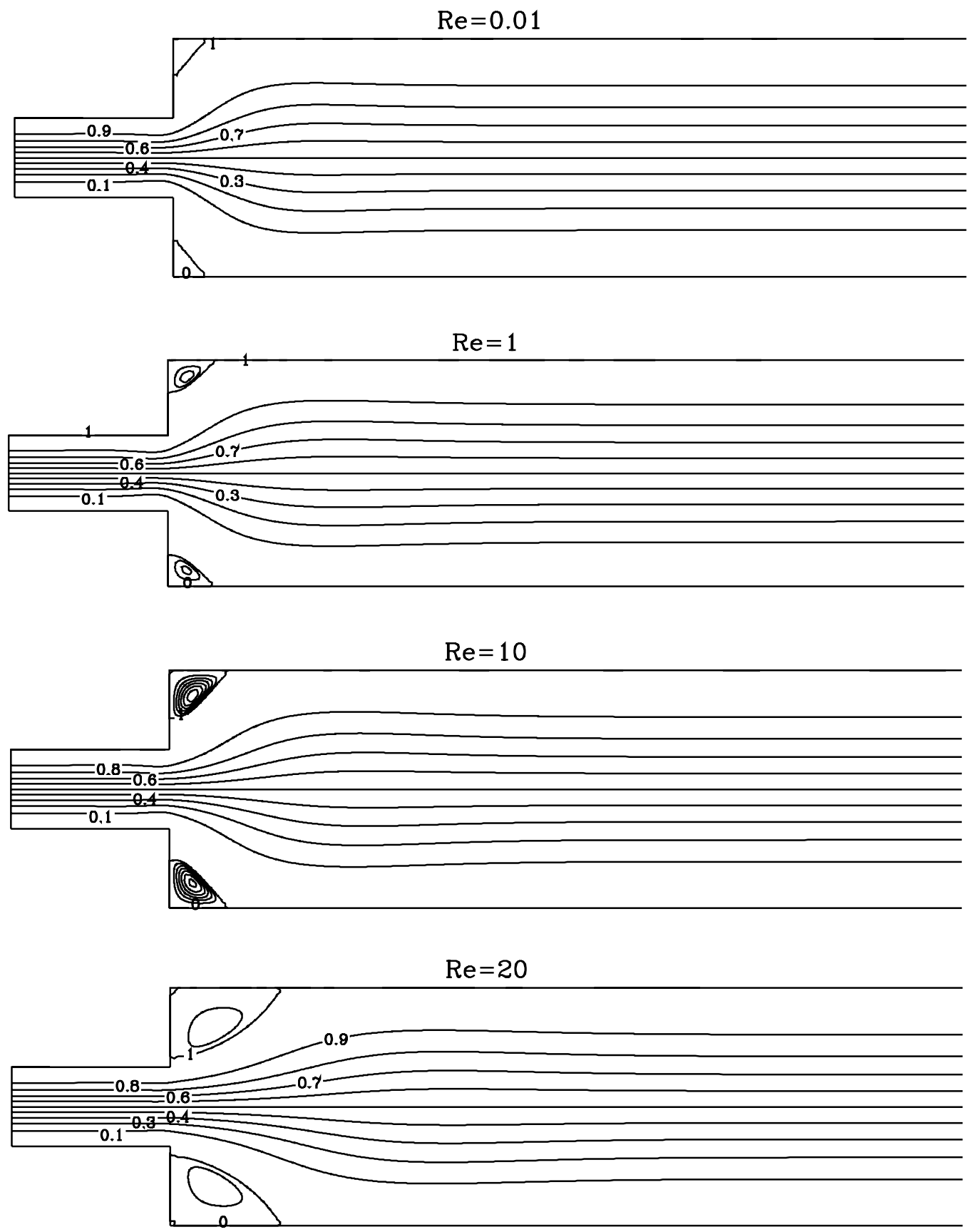

Fig. 14. Evolution with the Reynolds number of the flow patterns for the viscoelastic fluid case. 

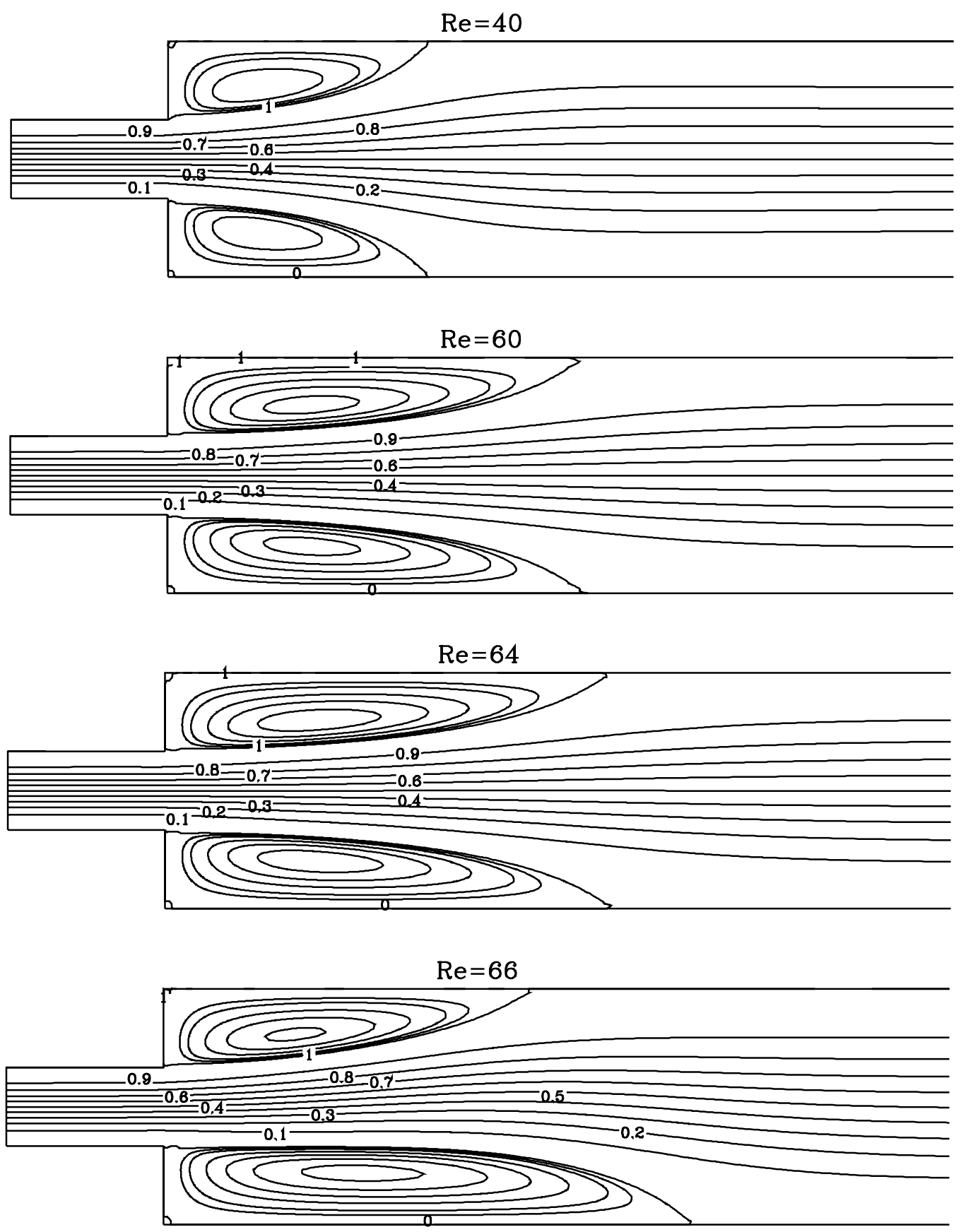

Fig. 14. (Continued) 

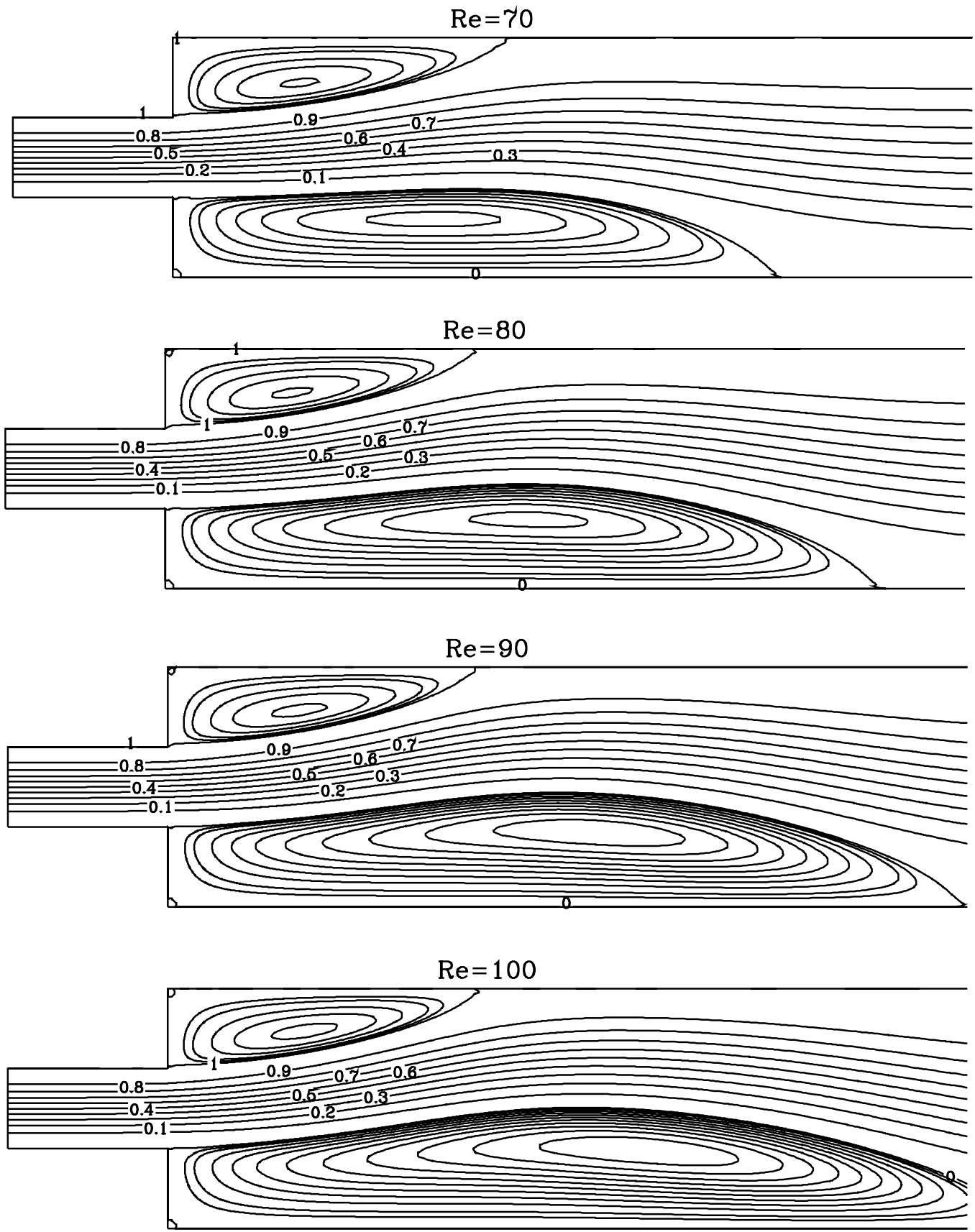

Fig. 14. (Continued). 


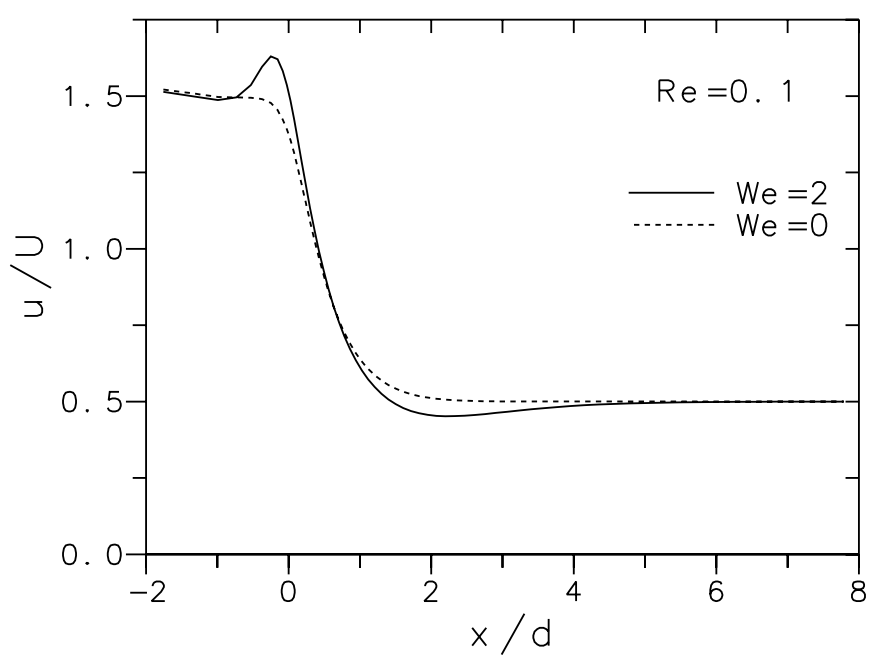

Fig. 15. Low Reynolds number case $(R e=0.1)$ : variation of streamwise velocity component along the central plane $(y=0)$, at $W e=0$ (Newtonian) and $W e=2$ (viscoelastic; $L^{2}=100$ and $\beta=0.5$ ).

Both these effects act to increase $C_{\mathrm{p}}$ of the FENE-MCR fluid compared with the Newtonian case.

It is emphasized that the $C_{\mathrm{p}}$ in Fig. 16 depends on the lengths of the upstream and downstream channels used in the simulations. A measure of the energy losses in the expansion which is independent of the assumed channel lengths is provided by the localized loss coefficient, defined as:

$$
C_{\mathrm{I}}=\frac{\Delta p-\Delta p_{\mathrm{F}}-\Delta p_{\mathrm{R}}}{(1 / 2) \rho U^{2}},
$$

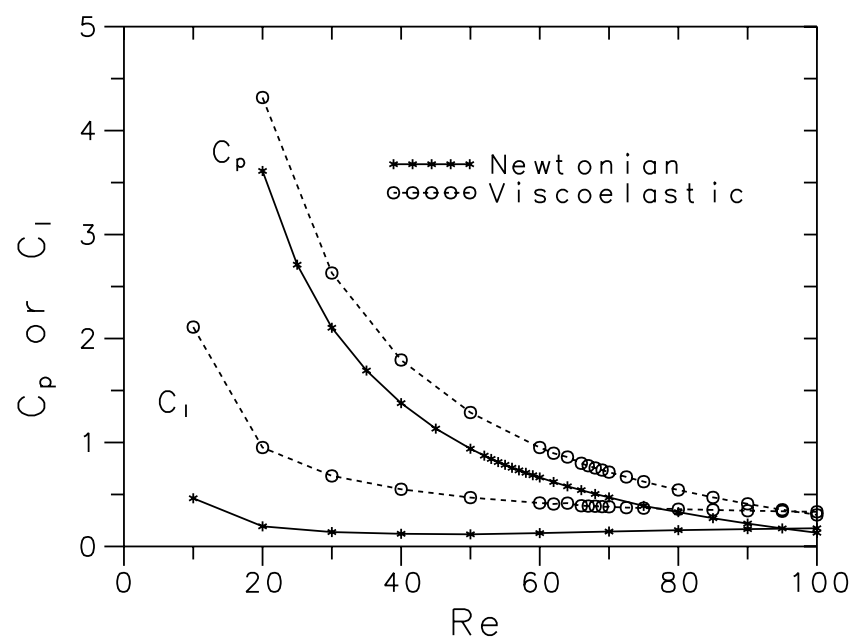

Fig. 16. Variation of the overall pressure drop and the localized loss coefficients with Reynolds number. Comparison of Newtonian against viscoelastic flow case $\left(L^{2}=100, \beta=0.5, W e=2\right)$. 


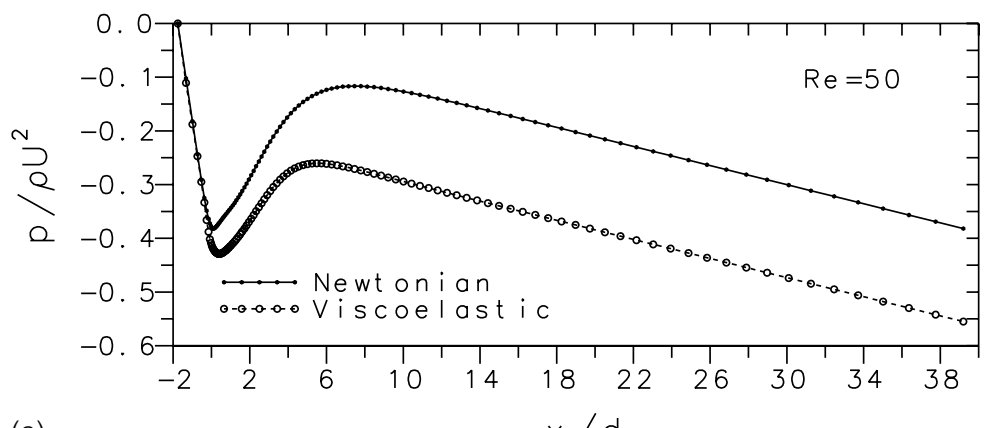

(a)

$\times / d$

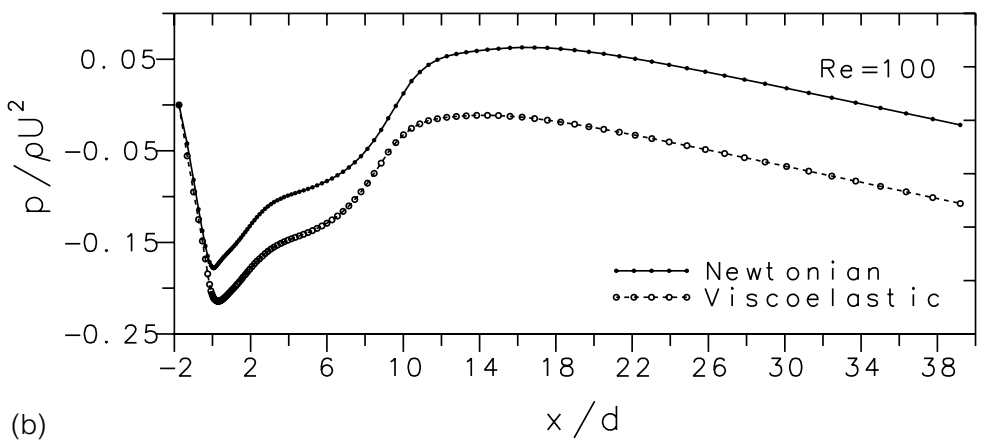

Fig. 17. Pressure profiles along central plane for Newtonian and viscoelastic fluids $\left(L^{2}=100, \beta=0.5, W e=2\right)$, at (a) $R e=50$ and (b) $R e=100$.

where the indices $\mathrm{F}$ and $\mathrm{R}$ denote fully-developed and reversible pressure differences, respectively. Due to pressure recovery in a reversible expansion flow, $\Delta p_{\mathrm{R}}$ is actually negative and may be easily evaluated from Bernoulli equation, giving $C_{\mathrm{R}}=-\left(1-\left(1 / E^{2}\right)\right)$. The fully-developed pressure drop coefficient, on the other hand, is given by contributions from the upstream and downstream channels as $C_{\mathrm{F}}=\left(\Delta p_{\mathrm{F}_{1}}+\right.$ $\left.\Delta p_{\mathrm{F}_{2}}\right) / 0.5 \rho U^{2}=2\left(f_{1} L_{1}+f_{2} L_{2} / E^{3}\right)$, where the $f^{\prime}$ 's are the Fanning friction factors, to be evaluated from the simulation results as $f_{1}=(\Delta P / \Delta X)_{1}$ and $f_{2}=(\Delta P / \Delta X)_{2} E^{3}$ (with: $P \equiv p / \rho U^{2} ; X \equiv x / d$ ). The important point here, for an accurate evaluation of $C_{\mathrm{I}}$ from the numerical results, is that the pressure gradients in the expressions for $f_{1}$ and $f_{2}$ need to be calculated in fully developed regions in the upstream and downstream channels. Otherwise, errors will accumulate leading to some deterioration in the accuracy of $C_{\mathrm{I}}$. We note that the loss coefficient of Eq. (27) is very similar to the Couette correction common in contraction flows of viscoelastic liquids, except for the inclusion of the reversible pressure-recovery term and the different normalisation.

In addition to the $C_{\mathrm{p}}$ variation, Fig. 16 also shows how the loss coefficient $C_{\mathrm{I}}$, calculated as just described, varies with $R e$. For the Newtonian case, longer inlet channel lengths have been used in extra calculations $\left(L_{1}=20\right)$ and so the $C_{\text {I }}$ results are accurate. For the viscoelastic case, the base value of $L_{1}=2$ does not enable a very precise evaluation of $f_{1}$ and the estimated accuracy of $C_{\mathrm{I}}$ varies between 2 and $10 \%$ (at low $R e$ ). These differences are not perceptible in a graph (except at low $R e$ in $\log -\log$ scales) and so Fig. 16 gives an adequate view of the variation of $C_{\mathrm{I}}$ versus $R e$. The loss coefficient is always positive, being higher for the viscoelastic case compared with the Newtonian (this is in contrast 
to findings in contraction flows). A change of slope is perceptible for the viscoelastic flow at the critical Reynolds number, while for the Newtonian liquid $C_{\mathrm{I}}$ attains a minimum at $R e=R e_{\mathrm{c}}$.

Because of this accuracy issue in the evaluation of the localized loss coefficient, we have decided to use also the flow resistance coefficient $f R e$ of Mishra and Jayaraman [10] as an (approximate) problem-independent measure of pressure loss. Under straight channel flow of length $l$ the definition of the Fanning friction factor is unambiguously related to the pressure drop, $\Delta p \equiv 4 f\left(l / D_{\mathrm{H}}\right)\left(\rho U^{2} / 2\right)$ where $D_{\mathrm{H}}$ is the hydraulic diameter equal to $D_{\mathrm{H}}=2 d$ for a plane channel of height $d$, and in this case we have a well-known theoretical result: $f \operatorname{Re}=12$ (with $R e \equiv \rho U d / \mu$; here $\mu \equiv \eta_{0}$ ). When an abrupt expansion is present an additional pressure drop occurs due to the irreversible energy losses in the recirculation zones that are generated, but we can still use an equivalent $f$ to measure these losses. A complication to using $f$ as defined above arises because there are different sizes and velocities in the upstream and downstream channel portions. It is however possible to write:

$$
\frac{d \Delta p_{1}}{\rho U_{1}^{2}}=f_{1} l_{1},
$$

and

$$
\frac{D \Delta p_{2}}{\rho U_{2}^{2}}=f_{2} l_{2}
$$

Now since $f \operatorname{Re}=12$ (or some other constant) and $\operatorname{Re}_{1}\left(\equiv \rho U_{1} d / \eta_{0}\right)=\operatorname{Re}_{2}\left(\equiv \rho U_{2} D / \eta_{0}\right)$ due to continuity, we have $f_{1}=f_{2} \equiv f$, and after summing (28) and (29) we obtain:

$$
f=\frac{\left(d \Delta p_{1} / \rho U_{1}^{2}\right)+\left(D \Delta p_{2} / \rho U_{2}^{2}\right)}{l_{1}+l_{2}},
$$

implying the following definition for $f R e$ :

$$
f R e=\operatorname{Re} \frac{\left(P_{1}-P_{2}\right)+\left(E^{3}-1\right)\left(P_{0}-P_{2}\right)}{L_{1}+L_{2}},
$$

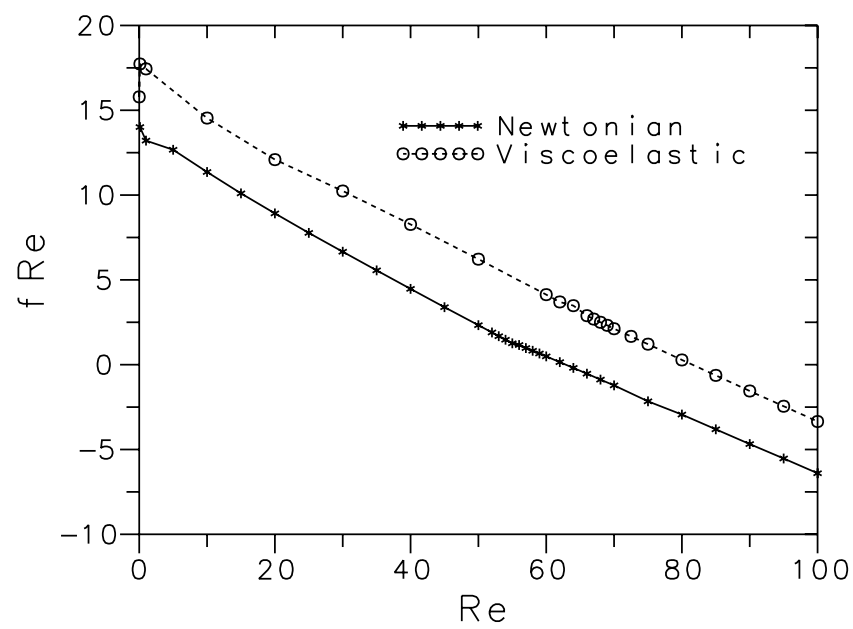

Fig. 18. Comparison of the flow resistance vs. Reynolds number variation for the Newtonian and the viscoelastic fluids $\left(L^{2}=100\right.$, $\beta=0.5, W e=2)$. 
where pressures are normalized as $P \equiv p / \rho U^{2}$ (note $U \equiv U_{1}$ ). In this expression, $P_{1}$ is the pressure at the inlet plane, $P_{0}$ is the pressure at the expansion plane $(x=0)$, and $P_{2}$ the pressure at the outlet plane. All these pressures were obtained from the simulation results, $f$ Re could then be calculated from Eq. (30) and is plotted as a function of the Reynolds number in Fig. 18. It is seen that the flow resistance decreases with $R e$, is higher for the viscoelastic fluid, and goes below zero beyond a certain $R e$ on account of pressure recovery. It is also apparent, although not so evident from the figure, that for the critical $R e_{\mathrm{c}}$ at which the asymmetry sets in, there is a change of slope in the $f R e$ versus $R e$ variation; $f R e$ versus $R e$ is more inclined for $R e<R e_{\mathrm{c}}$. Hence, we may conclude that the flow resistance, for both the Newtonian and viscoelastic fluids, would be less if the bifurcation had not occurred (an unstable state) and therefore energy losses are accentuated by the transition from stable symmetric to stable asymmetric states.

\section{Conclusions}

Numerical simulations of the flow of a FENE-MCR fluid through a planar expansion of expansion ratio 1:3 have been presented for a range of Reynolds numbers from 0 to 100 . Similar to the Newtonian fluid case, it has been shown that beyond a critical Reynolds number a pitch-fork-type bifurcation occurs for the viscoelastic fluid case, resulting in asymmetric vortex patterns. The effect of elasticity is to delay the onset of the bifurcation, the critical Reynolds number is $R e_{\mathrm{c}}=64$ for $W e=2, L^{2}=100$ and $\beta=0.5$, compared with $R e_{\mathrm{c}}=54$ for the Newtonian fluid in the same expansion, and to reduce the degree of flow asymmetry. Hence, after occurrence of the bifurcation, the size of the larger eddy tends to decrease for the elastic fluid, while the smaller eddy tends to increase; the eddy intensities, on the other hand, are always smaller for elastic fluids compared with inelastic.

A complete parametric study has been conducted, based on the numerical simulations, leading to the following conclusions regarding the separate effect of each individual parameter.

- Extensibility, through the constitutive parameter $L^{2}(=100-500)$, was seen to have a rather small effect upon the flow pattern (at least for low polymer concentrations).

- Polymer concentration, through the parameter $\beta=1 /(1+c)$, was seen to have a very strong effect: for $\beta=0.9$, at $R e=60$, the flow was asymmetric, but if $\beta$ was decreased to 0.5 (enhanced polymer concentration) the asymmetry was completely removed.

- Weissenberg number We, was seen to have some effect, mainly for the initial range of values $(W e \approx$ $2-5)$, with tendency to reduce the vortex asymmetry. This was correlated with the elongational viscosity behaviour of FENE-MCR.

- Inertia, through the Reynolds number $R e$, enabled construction of bifurcation diagrams, $D X \equiv X_{\mathrm{r} 2}-X_{\mathrm{r} 1}$ versus $R e$. These were seen to have similar form to the Newtonian case but were translated to the right, in the direction of higher $R e$.

The pressure losses are higher for the viscoelastic flow cases, compared with the Newtonian, and the bifurcation is accompanied by a step increase in pressure loss and flow resistance coefficients.

There are two lines one can envisage for future investigation. The most important is probably that of procuring experimental data for validating the present, or other studies with viscoelastic fluid flow through expansions. An effort along that direction has very recently been made by Poole and Escudier [34] (this work was published after submission of the present study) who considered turbulent flow of PAA $(R e=9100)$ in a 1:4 expansion. Hopefully, similar experiments in the laminar regime will be carried 
out in the near future. The second line of investigation is numerical and comes in the continuation of the present study: simulations at higher elasticity, for example, by lowering the value of $\beta$ (say $\beta=0.3$ ) and choosing some typical values of Weissenberg number (say $W e=10$ or 50) - the parameter to be varied will then be the Reynolds number. It will also be necessary to calculate accurate values of the pressure loss coefficient for the viscoelastic flow cases.

\section{Acknowledgements}

Financial support by FEDER and Fundação para a Ciência e Tecnologia (FCT), Portugal, under projects POCTI/EME/3771 1/2001 and POCTI/EQU/37699/01 is here recorded and gratefully acknowledged.

\section{References}

[1] D.V. Boger, K. Walters, Rheological Phenomena in Focus, Rheology Series, vol. 4, Elsevier, Amsterdam, 1993.

[2] S. Nigen, K. Walters, Viscoelastic contraction flows: comparison of axisymmetric and planar configurations, J. Non-Newtonian Fluid Mech. 102 (2002) 343-359.

[3] M.A. Alves, P.J. Oliveira, F.T. Pinho, Benchmark solutions for the flow of Oldroyd-B and PTT fluids in planar contractions, J. Non-Newtonian Fluid Mech. 110 (2003) 45-75.

[4] P. Townsend, K. Walters, Expansion flows of non-Newtonian liquids, Chem. Eng. Sci. 49 (1994) 749-763.

[5] A. Baloch, P. Townsend, M.F. Webster, On vortex development in viscoelastic expansion and contraction flows, J. Non-Newtonian Fluid Mech. 65 (1996) 133-149.

[6] A. Baloch, P. Townsend, M.F. Webster, On two- and three-dimensional expansion flows, Comput. Fluids 24 (1995) $863-882$.

[7] M.S. Darwish, J.R. Whiteman, M.J. Bevis, Numerical modelling of viscoleastic liquids using a finite-volume method, J. Non-Newtonian Fluid Mech. 45 (1992) 311-337.

[8] K.A. Missirlis, D. Assimacopoulos, E. Mitsoulis, A finite volume approach in the simulation of viscoelastic expansion flows, J. Non-Newtonian Fluid Mech. 78 (1998) 91-118.

[9] R.J. Poole, M.P. Escudier, Turbulent flow of non-Newtonian liquids over a backward-facing step. Part II. Viscoelastic and shear-thinning liquids, J. Non-Newtonian Fluid Mech. 109 (2003) 193-230.

[10] S. Mishra, K. Jayaraman, Asymmetric flows in planar symmetric channels with large expansion ratio, Int. J. Numer. Methods Fluids 38 (2002) 945-962.

[11] R.M. Fearn, T. Mullin, K.A. Cliffe, Nonlinear flow phenomena in a symmetric sudden expansion, J. Fluid Mech. 211 (1990) 595-608.

[12] D. Drikakis, Bifurcation phenomena in incompressible sudden expansion flows, Phys. Fluids 9 (1997) 76-86.

[13] T. Hawa, Z. Rusak, The dynamics of a laminar flow in a symmetric channel with a sudden expansion, J. Fluid Mech. 436 (2001) 283-320.

[14] M.D. Chilcott, J.M. Rallison, Creeping flow of dilute polymer solutions past cylinders and spheres, J. Non-Newtonian Fluid Mech. 29 (1988) 381-432.

[15] J. Remmelgas, P. Singh, L.G. Leal, Computational studies of nonlinear elastic dumbbell models of Boger fluids in a cross-slot flow, J. Non-Newtonian Fluid Mech. 88 (1999) 31-61.

[16] R.B. Bird, P.J. Dotson, N.L. Johnson, Polymer solution rheology based on a finitely extensible bead-spring chain model, J. Non-Newtonian Fluid Mech. 7 (1980) 213-235.

[17] R.B. Bird, O. Hassager, R.C. Armstrong, C.F. Curtiss, Dynamics of Polymer Liquids, vol. 2, Wiley, New York, 1977.

[18] R.B. Bird, J.M. Wiest, Constitutive equations for polymeric liquids, Annu. Rev. Fluid Mech. 27 (1995) 169-193.

[19] P.J. Coates, R.C. Armstrong, R.A. Brown, Calculation of steady-state viscoelastic flow through axisymmetric contractions with the EEME formulation, J. Non-Newtonian Fluid Mech. 42 (1992) 141-188.

[20] P.J. Oliveira, Method for time-dependent simulations of viscoelastic flows: vortex shedding behind cylinder, J. Non-Newtonian Fluid Mech. 101 (2001) 113-137. 
[21] P.J. Oliveira, F.T. Pinho, G.A. Pinto, Numerical simulation of non-linear elastic flows with a general collocated finite-volume method, J. Non-Newtonian Fluid Mech. 79 (1998) 1-43.

[22] C.M. Rhie, W.L. Chow, A numerical study of the turbulent flow past an airfoil with trailing edge separation, AIAA J. 21 (1983) 1525-1532.

[23] P.J. Oliveira, F.T. Pinho, Numerical procedure for the computation of fluid flow with arbitrary stress-strain relationships, Numerical Heat Trans. B 35 (1999) 295-315.

[24] M.A. Alves, P.J. Oliveira, F.T. Pinho, A convergent and universally bounded interpolation scheme for the treatment of advection, Int. J. Numer. Methods Fluids 41 (2003) 47-75.

[25] J.P. Van Doormaal, G.D. Raithby, Enhancements of the SIMPLE method for predicting incompressible fluid flows, Numerical Heat Transfer 7 (1984) 147-163.

[26] R.I. Issa, P.J. Oliveira, Numerical prediction of phase separation in two-phase flow through T-junctions, Comput Fluids 23 (1994) 347-372.

[27] P.J. Oliveira, F.T. Pinho, Pressure drop coefficient of laminar Newtonian flow in axisymmetric sudden expansions, Int. J. Heat Fluid Flow 18 (1997) 518-529.

[28] M.A. Alves, F.T. Pinho, P.J. Oliveira, Effect of a high-resolution differencing scheme on finite-volume predictions of viscoelastic flows, J. Non-Newtonian Fluid Mech. 93 (2000) 287-314.

[29] F. Durst, J.C.F. Pereira, C. Tropea, The plane symmetric sudden-expansion flow at low Reynolds numbers, J. Fluid Mech. 248 (1993) 567-581.

[30] S.R.N. De Zilwa, L. Khezzar, J.H. Whitelaw, Flows through plane sudden-expansions, Int. J Numer. Methods Fluids 32 (2000) 313-329.

[31] B. Purnode, M.J. Crochet, Polymer solution characterization with the FENE-P model, J. Non-Newtonian Fluid Mech. 77 (1998) 1-20.

[32] S.V. Patankar, Numerical Heat Transfer and Fluid Flow, Hemisphere, Washington, 1980.

[33] A.W. Liu, D.E. Bornside, R.C. Armstrong, R.A. Bronw, Viscoelastic flow of polymer solutions around a periodic, linear array of cylinders: comparisons of predictions for microstructure and flow fields, J. Non-Newtonian Fluid Mech. 77 (1998) 153-190.

[34] R.J. Poole, M.P. Escudier, Turbulent flow of a viscoelastic shear-thinning liquid through a plane sudden expansion of modest aspect ratio, J. Non-Newtonian Fluid Mech. 112 (2003) 1-26. 